\title{
Motion of a Spot in a Reaction Diffusion System under the Influence of Chemotaxis
}

\author{
Satoshi Kawaguchi $(\mathbb{D}$ \\ Department of Complex and Intelligent Systems, School of Systems Information Science, Future University Hakodate, \\ Hakodate 041-8655, Japan \\ Correspondence should be addressed to Satoshi Kawaguchi; satoshi@fun.ac.jp
}

Received 7 December 2017; Accepted 29 March 2018; Published 21 May 2018

Academic Editor: Christos Volos

Copyright (C) 2018 Satoshi Kawaguchi. This is an open access article distributed under the Creative Commons Attribution License, which permits unrestricted use, distribution, and reproduction in any medium, provided the original work is properly cited.

\begin{abstract}
We consider the motion of a spot under the influence of chemotaxis. We propose a two-component reaction diffusion system with a global coupling term and a Keller-Segel type chemotaxis term. For the system, we derive the equation of motion of the spot and the time evolution equation of the tensors. We show the existence of an upper limit for the velocity and a critical intensity for the chemotaxis, over which there is no circular motion. The chemotaxis suppresses the range of velocity for the circular motion. This braking effect on velocity originates from the refractory period behind the rear interface of the spot and the negative chemotactic velocity. The physical interpretation of the results and its plausibility are discussed.
\end{abstract}

\section{Introduction}

The behaviors of artificial and biological microswimmers such as oil droplets, bimetallic nanorods, catalytic Janus colloids, liposomes, flagellated bacteria, and Volvox have attracted widespread attention [1]. Under certain circumstances, some of these microswimmers are self-propelled particles, the mobility mechanism of which has been intensively studied $[2,3]$. The motion of oil droplets, especially, has been studied in well-controlled experimental facilities with sufficient reproducibility. Although symmetric droplets cannot move in the absence of external force, the Marangoni effect can cause motion in the presence of an inhomogeneous chemical substance outside the droplet or a temperature gradient along the surface [4-6]. Numerical simulations and theoretical results support this mechanism and the existence of straight, circular, and complicated motions of droplets $[7,8]$, and experimental results qualitatively agree with the numerical results [9-12]. Droplet motion has also been the subject of a review article [13, 14].

In a two-dimensional reaction diffusion (RD) system, the droplet is often referred to as a spot solution. In order to systematically describe the motion of spots in an RD system, the time evolution equation of the spot was derived and the mechanism of elastic collision of moving spots was clarified in a previous study [15]. This study was extended by studies on the drift and rotation bifurcations of spot solutions in RD systems $[16,17]$. In order to describe the deformations of the spot, tensors were introduced. The bifurcation diagram of the spot suggested that, with increasing velocity of the spot, rotation bifurcation occurred causing the straight motion to become destabilized into circular motion.

In addition to the Marangoni effect, which plays an important role in the motion of oil droplets, chemotaxis is an important property of cell migration; it is important in mass transfer and immunological response in biology. In inflammatory response, the neutrophils among blood cells have a remarkable migration potency (chemotaxis) and can change their form by generating pseudopods toward the antigen. In biophylaxis, several chemokines (chemoattractants) are released from the macrophages and mast cells. Then other immunocompetent cells (neutrophils) respond to the gradient of the chemoattractant. Consequently, the immunocompetent cells move unidirectionally to the source point of the antigen [18]. 
The mathematical model for chemotaxis was first proposed by Keller and Segel [19], wherein the gradient of the chemoattractant was taken into consideration for the flow of amoeba. Neutrophil migration was considered with a Keller-Segel type chemotaxis term in [20, 21]. In these studies, the Cahn-Hilliard $(\mathrm{CH})$ equation was employed, and the kinematic properties and morphological changes of the crawling cell distribution were shown. In addition to the chemotaxis of the neutrophil, cancer cell invasion under haptotaxis was modeled by the $\mathrm{CH}$ equation $[22,23]$. The haptotactic response of cancer cells is described by the gradient of the haptoattractant. However, in the above studies, the gradients of chemoattractant and haptoattractant are assumed to be constant; there is no feedback between the cells and these chemical substances.

As described above, with the recent increase in importance of chemotaxis in biology, medicine, and cytoengineering [24-27], many experimental and theoretical studies have been performed. Although there are model systems for the cell density and concentration of chemotactic substances, no mathematical analysis has been reported on the motion of the cell. Inspired by these points, we first propose an $\mathrm{RD}$ system including a naive Keller-Segel type chemotaxis term. The system is autonomous, the spot secretes a chemotactic substance, and the motion of the spot is influenced by it. For the proposed $\mathrm{RD}$ system, we apply the method reported in [16] to derive the equation of motion of the spot and time evolution equation of the tensors. Based on these equations, we study the bifurcation from straight motion to circular motion as well as the upper limit of the velocity of circular motion. In order to verify the theoretical result, we perform numerical simulations for the tensor model. The physical meaning and validity of the results are discussed.

\section{Model Equation}

We first consider the following three-component RD system with an activator $u$, a chemotactic substance $v$, and an inhibitor $w$ :

$$
\begin{gathered}
\tau \epsilon \frac{\partial u}{\partial t}=\epsilon^{2} \nabla^{2} u-\epsilon \nabla \cdot(u \nabla \chi(v)) \\
+H(u-p(w))-u-v, \\
\frac{\partial v}{\partial t}=\nabla^{2} v+u-\mu v, \\
T \frac{\partial w}{\partial t}=D \nabla^{2} w+u+v-w-W,
\end{gathered}
$$

where $p(w)=p_{0}+\alpha w, p_{0}, \alpha, \tau, \epsilon, \mu, T, D$, and $W$ are positive constants, and $H(z)$ is a step function satisfying $H=0$ for $z<0$ and $H=1$ for $z>0$. Throughout this study, we consider the system in a two-dimensional space, with $\nabla=\left(\partial_{x}, \partial_{y}\right)$ and $\epsilon \ll 1$. We choose $\mu$ such that the system is monostable. Here, we fix $\mu=0.3$. In the above excitable system, there are two stationary states: a rest state and an excited state. The rest state is $(u, v, w)=(0,0,-W)$, and the excited state has spatially nonuniform values of $u, v$, and $w$. Between the rest and excited states, there appear boundary layers with thickness $O(\epsilon)$, connecting the two different states.

When the second term on the right hand side of (1) is absent, (1)-(3) describe an RD system with one activator and two inhibitors, which was studied in [28]. In that system, when $\tau$ is large, the localized domain (motionless spot solution) of an activator appears. With decreasing $\tau$, the motionless spot is destabilized through static bifurcation or oscillatory bifurcation; however, when $T$ is small and $D$ and $\alpha$ are large, these bifurcations are suppressed by $w$. When $\tau$ is small, the motionless spot is primarily destabilized through translational bifurcation, causing the spot to move.

In the presence of the second term on the right hand side of (1), the moving spot is influenced by the chemotaxis. A system similar to that described by (1)-(3), but with bistability, was studied in [29]. In that system, the nonlinear term in (1) was replaced by $H(u-p(w))-u$, and a front solution was obtained. Furthermore, maze patterns and branching from a front solution were observed. The stability analyses of the spot and front solutions were conducted by applying the singular perturbation method [30].

The time evolution equation for $u$ is obtained using the conservation equation. The diffusion term is derived from $-\nabla \cdot \mathbf{J}$, where the flux $\mathbf{J}$ is the sum of the normal diffusion (random motility) term $\mathbf{J}_{\mathrm{d}}$ and the chemotaxis term $\mathbf{J}_{\mathrm{c}}$. That is, $\mathbf{J}=\mathbf{J}_{\mathrm{d}}+\mathbf{J}_{\mathrm{c}}$, where $\mathbf{J}_{\mathrm{d}}=-\epsilon^{2} \nabla u$ and $\mathbf{J}_{\mathrm{c}}=\epsilon u \nabla \chi(v)$. It should be noted that the signs of these fluxes are different. The sign of $\mathbf{J}_{\mathrm{c}}$ suggests that the chemotaxis term provides a negative diffusion effect, which suppresses the expansion of $u$. The second term on the right hand side of (1) is the Keller-Segel type chemotaxis term; we express the chemotactic sensitivity function $\chi$ as $\chi(v)=k_{c} \chi_{0}(v)$, where $\chi_{0}(v)=f_{0} \theta v^{2} /\left(v^{2}+\theta^{2}\right)$ with $\theta=1.0$. In order to satisfy the condition $\max \left|d \chi_{0} / d v\right|=$ 1 , we choose $f_{0}=8 \sqrt{3} / 9$. We call $k_{c}$ the intensity of chemotaxis [31].

In (3), $T$ and $D$ represent the relaxation time and diffusion constant of $w$, respectively. Let us consider a situation in which $w$ plays the role of feedback to suppress the static bifurcation and oscillatory bifurcation. For the rapid feedback mechanism, $T$ and $D$ must be small and large, respectively. In the limits $T \rightarrow 0$ and $D \rightarrow \infty, w$ becomes a time-dependent but spatially independent variable, which is denoted by $\langle w(t)\rangle$ :

$$
\langle w(t)\rangle=\frac{1}{\Omega_{0}} \int d \mathbf{r} w=\frac{1}{\Omega_{0}} \int d \mathbf{r}(u+v)-W
$$

where $\Omega_{0}$ is the area of the entire system. Replacing $w$ in $p(w)$ by $\langle w(t)\rangle, p(w)$ becomes a global coupling term. In the case where $T$ is very small and $D$ is very large, we reduce the threecomponent $\mathrm{RD}$ system to the following two-component $\mathrm{RD}$ system with a global coupling term:

$$
\begin{aligned}
\tau \epsilon \frac{\partial u}{\partial t} & =\epsilon^{2} \nabla^{2} u-\epsilon \nabla \cdot(u \nabla \chi(v))+f\{u, v\}-v, \\
\frac{\partial v}{\partial t} & =\nabla^{2} v+u-\mu v
\end{aligned}
$$


where

$$
f\{u, v\}=-u+H(u-p\{u, v\}) .
$$

The functional $p\{u, v\}$ represents a global coupling term given by

$$
p\{u, v\}=p_{0}+\alpha\left[\int d \mathbf{r}(u+v)-W\right]
$$

where the integral is over the entire domain, and $\alpha$ and $W$ are rescaled to absorb $\Omega_{0}: \alpha^{\prime}=\alpha / \Omega_{0}$ and $W^{\prime}=W \Omega_{0}$, and the primes are dropped. $\alpha$ corresponds to the intensity of the global coupling, and the value of $p_{0}$ is chosen as $p_{0}=$ 0.275 . Hereinafter, we consider the above two-component RD system to be described by (5) and (6).

In the absence of chemotaxis, (5) and (6) describe the same system proposed by Krischer and Mikhailov [32]. This system had an activator and an inhibitor, and, for large $\tau$, the motionless spot (localized particle-like structure) in two dimensions was stable. With decreasing $\tau$ under a large $\alpha$, it was shown that the system had a stable moving spot. In order to understand intuitively the bifurcation from the motionless spot to the moving spot, we consider the limit $\epsilon \rightarrow 0$. In the limit $\epsilon \rightarrow 0$, the boundary layer of $u$ becomes an interface, as shown in Figure 1. The location of the interface is defined by the condition $u(\mathbf{r}, t)=p$. In this limit, $u$ and $v$ satisfy the relation $(u+v)=1$ (inside the domain) and 0 (outside the domain), which is obtained from (5). In the limits of $\alpha \rightarrow \infty$ and $\epsilon \rightarrow 0$, the area of the spot is conserved; using (8), we obtain

$$
\int d \mathbf{r}(u+v)=W
$$

This restriction prohibits the expansion and oscillation of the spot; the translational bifurcation firstly occurs with decreasing $\tau$. Even for finite values of a large $\alpha$ and small $\epsilon$, the area of the spot is approximately conserved by the feedback mechanism. This supports the existence of a moving spot for small $\tau$ with large $\alpha$. In contrast, for small $\alpha$, the static bifurcation or oscillatory bifurcation firstly occurs with decreasing $\tau$, and the motionless spot is destabilized to form an expanding wave or is disintegrated by unstable oscillation.

Although the bifurcation from a motionless spot to a straight moving spot and the collision of two moving spots were studied in [32], other types of moving spot were not studied. Recently, the bifurcation of the spot from straight motion to circular motion was theoretically analyzed for the system described by (5) and (6) without the chemotaxis term [16]. The result suggested that there was a critical value $\tau_{c}$ for the bifurcation of stationary straight motion. Although the straight motion was stable for $\tau>\tau_{c}$, it was destabilized by rotation (straight-circular-motion) bifurcation for $\tau \leq \tau_{c}$. In other words, the straight motion was destabilized into circular motion with increasing velocity.

In this study, we assume that the localized domain (spot) of an activator exists under global feedback in the system described by (5) and (6). In the system, the chemotactic substance is secreted from inside the spot, and the motion of the spot is influenced by the chemotaxis. In order to study the

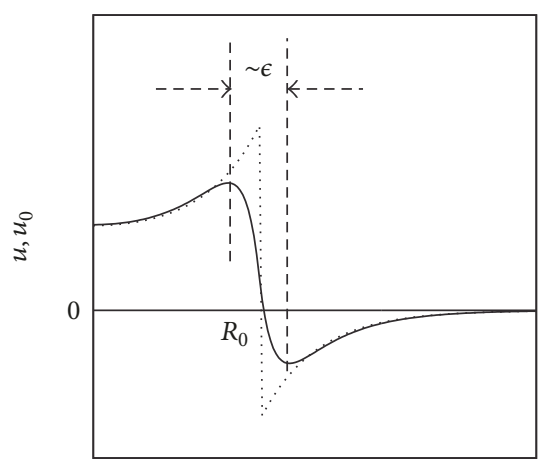

$|\mathbf{r}|$

FIgURE 1: Boundary layer and interface. The solid and dotted curves correspond to $u(\mathbf{r}, t)$ and $u_{0}(\mathbf{r}, t)=\lim _{\epsilon \rightarrow 0} u(\mathbf{r}, t)$ along a radial direction, respectively, that is, $u(|\mathbf{r}|, t)$ and $u_{0}(|\mathbf{r}|, t)$. In the finite $\epsilon, u(|\mathbf{r}|, t)$ has a boundary layer around $|\mathbf{r}| \sim R_{0}$ with the thickness of $\epsilon$, where $R_{0}$ is determined by the condition $u\left(|\mathbf{r}|=R_{0}\right)=p \cdot u_{0}(|\mathbf{r}|, t)$ does not have a boundary layer but has a sharp interface at $|\mathbf{r}|=R_{0}$.

influence of chemotaxis on the motion of a spot, we apply the technique reported in [16] to the system described by (5) and (6). For this purpose, we first derive the radially symmetric equilibrium solution of (5) and (6) in the limit $\epsilon \rightarrow 0$. In this limit, (6) becomes

$$
\frac{\partial v}{\partial t}=\nabla^{2} v+H(u-p)-\beta v
$$

where $\beta=1+\mu$. Setting $\partial v / \partial t=0$ in (10), (10) becomes

$$
\left(\frac{d^{2}}{d r^{2}}+\frac{1}{r} \frac{d}{d r}\right) v-\beta v+H\left(R_{0}-r\right)=0,
$$

where $r=|\mathbf{r}|$ and $R_{0}$ is the equilibrium radius of the symmetric spot. We impose the boundary conditions on $v(r)$ as

$$
\begin{aligned}
\frac{d v(0)}{d r} & =0, \\
v\left(R_{0}+\right) & =v\left(R_{0^{-}}\right), \\
\frac{d v\left(R_{0}+\right)}{d r} & =\frac{d v\left(R_{0}-\right)}{d r}, \\
v(\infty) & =0 .
\end{aligned}
$$

The radially symmetric equilibrium solution of (10) in two dimensions is given by

$$
\begin{aligned}
& \bar{v}(r) \\
& \quad= \begin{cases}\frac{1}{\beta}-\frac{R_{0}}{\sqrt{\beta}} K_{1}\left(\sqrt{\beta} R_{0}\right) I_{0}(\sqrt{\beta} r), & 0<r<R_{0}, \\
\frac{R_{0}}{\sqrt{\beta}} I_{1}\left(\sqrt{\beta} R_{0}\right) K_{0}(\sqrt{\beta} r), & R_{0}<r<\infty,\end{cases}
\end{aligned}
$$

where $I_{n}$ and $K_{n}$ are the modified Bessel functions of the first and second kinds of order $n$, respectively. The corresponding 
solution of $u(r)$ is given by $\bar{u}(r)=H\left(R_{0}-r\right)-\bar{v}(r)$. The above equilibrium solution is employed to study the motion of the spot in the following sections.

\section{Equation of Motion of the Interface}

As $\epsilon$ is small, the width of the boundary layer is small, and, therefore, the value of $u$ changes sharply while that of $v$ changes smoothly around the boundary layer. The velocity of the flat interface is a function of the value of $v$ on the interface. In order to consider the dynamics of the spot in the system, we derive the equation of motion of the interface. The time evolution equation of the interface is described $[16,30,33]$ by

$$
\tau V=\tau C(h)+\epsilon \kappa+L,
$$

where $C(h)$ is the velocity of a flat interface; $\kappa$ is the curvature of the interface, the sign of which is chosen such that it is positive when the center of the curvature is outside of the excited domain; and $h$ is the value of $v$ on the interface. $V$ is the normal component of the velocity, and $L$ is the Lagrange multiplier for the constraint of domain area conservation (9). For the moving spot, this condition corresponds to

$$
\int d \omega V(\omega)=0
$$

where $d \omega$ is the infinitesimal length of the interface and the integral is carried over the entire interface.

We first consider the velocity of the interface in one dimension in (5) and (6). With the detailed derivations given in Appendix A, the velocity $\tau C(h)$ is obtained as

$$
\begin{aligned}
\tau C(h)= & \frac{2(1 / 2-h-p)}{[(h+p)(1-h-p)]^{1 / 2}} \\
& +\left.\left.\left(\frac{d \chi}{d v}\right)\right|_{i}\left(\frac{d v}{d x}\right)\right|_{i},
\end{aligned}
$$

where $p$ is a global coupling term (8). $\tau C(h)$ consists of two components: the velocity of the traveling front solution in one dimension and the chemotactic velocity.

In two dimensions, the velocity of a flat interface directed along $\mathbf{n}$ is given by

$$
\begin{aligned}
\tau C(h) & =\frac{2(1 / 2-h-p)}{[(h+p)(1-h-p)]^{1 / 2}}+\left.k_{c} \chi_{0}^{\prime}(v)\left(\frac{d v}{d \mathbf{n}}\right)\right|_{i} \\
& \equiv P(h)+\left.Q(h)(\mathbf{n} \cdot \nabla) v\right|_{i},
\end{aligned}
$$

where $\chi_{0}^{\prime}(v)=d \chi_{0}(v) / d v$ and $\left.A\right|_{i}$ represents the value of function $A$ evaluated on the interface. $\mathbf{n}$ is a unit normal vector on the interface, and $(d v / d \mathbf{n})$ is a normal derivative.

When the motion of the interface of the spot is slow compared with the relaxation rate of the chemotactic substance, the left hand side of (10), $(\partial v / \partial t)$, is small. In this case, we deal with the time derivative of $v$ in (10) as a perturbation.
The asymptotic solution of (10) is written by perturbation expansion using the Green function $G$ as

$$
\begin{aligned}
v(\mathbf{r}, \mathrm{t})= & G H-G G \frac{\partial H}{\partial t}+G G G \frac{\partial^{2} H}{\partial t^{2}}-G G G G \frac{\partial^{3} H}{\partial t^{3}} \\
& +\cdots,
\end{aligned}
$$

where $G$ is Green's function satisfying the equation

$$
\left(\nabla^{2}-\beta\right) G\left(\mathbf{r}-\mathbf{r}^{\prime}\right)=-\delta\left(\mathbf{r}-\mathbf{r}^{\prime}\right)
$$

In (18), $G H$ represents the integral

$$
\begin{aligned}
G H & =\int d \mathbf{r}_{1} G\left(\mathbf{r}-\mathbf{r}_{1}\right) H\left(\mathbf{r}_{1}, t\right) \\
G G \frac{\partial H}{\partial t} & =\int d \mathbf{r}_{1} d \mathbf{r}_{2} G\left(\mathbf{r}-\mathbf{r}_{1}\right) G\left(\mathbf{r}_{1}-\mathbf{r}_{2}\right) \frac{\partial H\left(\mathbf{r}_{2}, t\right)}{\partial t}, \\
\cdots & =\cdots,
\end{aligned}
$$

for brevity. In this study, we consider the situation in which the motionless spot is destabilized supercritically and the spot moves with an arbitrarily small velocity. Then, we can safely apply the perturbative expansion of $v$ in terms of $G$ as in (18).

\section{Deformed Spot Dynamics}

In this section, we derive the equation of motion of the spot. In order to describe the deformations of the spot, tensors are introduced. The tensors depend on time, and the time evolution equation of the tensors are derived following [16].

4.1. Description of Deformed Spot. We firstly describe the position and velocity of a deformed spot. The motion of the spot consists of two components: the motion of the center of gravity and the motion of the interface relative to the center of gravity. The center of gravity is denoted by $\rho$, and the velocity of the center of gravity is given by

$$
\frac{d \boldsymbol{\rho}}{d t}=\frac{1}{\Omega} \int d \omega V(\omega) \mathbf{R}(\omega) \equiv \mathbf{v},
$$

where $\Omega$ is the area of the spot and $\mathbf{R}(\omega)$ is a position vector measured from the center of gravity. We consider the case that $\mathbf{R}(\omega)$ is a single valued function of $\phi_{r}$, which is given as

$$
\mathbf{R}(\omega)=R\left(\phi_{r}\right) \mathbf{e}_{r},
$$

where $\mathbf{e}_{r}$ is a radial unit vector and $R\left(\phi_{r}\right)$ is the distance between the center of gravity and a point on the interface, which is directed to an angle $\phi_{r}$ with respect to the $x$ axis. Using the definition of $\mathbf{R}(\omega), d \omega$ is given by

$$
d \omega=\left|\frac{d \mathbf{R}}{d \phi_{r}}\right| d \phi_{r}=\sqrt{R^{2}+R^{\prime 2}} d \phi_{r},
$$

where $R^{\prime}=d R\left(\phi_{r}\right) / d \phi_{r}$. For the deformed spot, the radius is given by

$$
R\left(\phi_{r}\right)=R_{0}+\delta R\left(\phi_{r}, t\right)
$$


with

$$
\delta R\left(\phi_{r}, t\right)=\sum_{n=-\infty}^{\infty} c_{n}(t) e^{\mathrm{in} \phi_{r}},
$$

where $\mathrm{i}=\sqrt{-1}, R_{0}$ is the equilibrium radius of the symmetric spot, and $\delta R$ corresponds to the deviation. In the expansion of $\delta R, c_{+n}$ corresponds to a $(2 \pi / n)$-periodic deformation. According to the area conservation equation (9), $c_{0}=0$. In addition, we exclude the terms of $n= \pm 1$ because the translational motion of the spot is incorporated in $\rho$. Thus, we set $c_{ \pm 1}=0$. Using (24), the curvature $\kappa$ and the normal component of the velocity $V$ are given up to the first order of the deviations as

$$
\begin{aligned}
\kappa\left(\phi_{r}, t\right) & =-\frac{1}{R_{0}}-\frac{1}{R_{0}^{2}} \sum_{n=-\infty}^{\infty}\left(n^{2}-1\right) c_{n}(t) e^{\mathrm{i} n \phi_{r}}, \\
V\left(\phi_{r}, t\right) & =\mathbf{v} \cdot \mathbf{n}+\sum_{n=-\infty}^{\infty} \dot{c}_{n}(t) e^{\mathrm{i} n \phi_{r}}
\end{aligned}
$$

respectively, where the overdot denotes the time derivative.

For a general function $A(\mathbf{r}, t)$, the Fourier transformation and its reverse transformation in two dimensions are defined as

$$
\begin{aligned}
A_{\mathbf{q}}(t) & =\int d \mathbf{r} A(\mathbf{r}, t) e^{-\mathrm{i} \mathbf{q} \cdot \mathbf{r}}, \\
A(\mathbf{r}, t) & =\frac{1}{(2 \pi)^{2}} \int d \mathbf{q} A_{\mathbf{q}}(t) e^{\mathrm{i} \mathbf{q} \cdot \mathbf{r}} \equiv \int_{\mathbf{q}} A_{\mathbf{q}}(t) e^{\mathrm{i} \mathbf{q} \cdot \mathbf{r}},
\end{aligned}
$$

respectively, where $\int_{\mathbf{q}}=\int d \mathbf{q} /(2 \pi)^{2}$ for brevity. For an isolated domain that forms a single loop without crossing, the step function $H(u-p)=H(R-|\mathbf{r}-\boldsymbol{\rho}|)$ is transformed by the Fourier transformation as

$$
H_{\mathbf{q}}=\int d \mathbf{r} H(u-p) e^{-\mathbf{i} \mathbf{q} \cdot \mathbf{r}}=\int_{|\mathbf{r}-\boldsymbol{\rho}|<R} d \mathbf{r} e^{-\mathbf{i} \mathbf{q} \cdot \mathbf{r}} .
$$

Substituting (24) into (30), we obtain the expansion of $H_{\mathbf{q}}$ up to the first order of $\delta R$ as

$$
\begin{aligned}
H_{\mathbf{q}} & =H_{\mathbf{q}}^{(0)}+H_{\mathbf{q}}^{(1)}, \\
H_{\mathbf{q}}^{(0)} & =\frac{2 \pi R_{0}}{q} J_{1}\left(q R_{0}\right), \\
H_{\mathbf{q}}^{(1)} & =2 \pi R_{0} \sum_{n} c_{n}(t) \mathrm{i}^{-n} e^{\mathrm{i} n \phi_{q}} J_{n}\left(q R_{0}\right),
\end{aligned}
$$

where $q=|\mathbf{q}|, \phi_{q}$ is the angle between $\mathbf{q}$ and the $x$-axis, and $J_{n}$ is a Bessel function of the first kind of order $n$.

4.2. Equation of Motion of a Spot. We derive the equation of motion of a spot from (14). $\tau C$ is given as functions of $h$ and $\widetilde{h}$ by (17), in which we used the notation $\widetilde{h}=$ $\left.(d v / d \mathbf{n})\right|_{i}=\left.(\mathbf{n} \cdot \nabla) v\right|_{i}$. When the velocity of the spot is small, the deformation of the circle is small. For this situation, we derive the deviations of $h$ and $\widetilde{h}$ from their stationary values as a power series of $\tau C$. Using (18), we first expand $h$ and $\widetilde{h}$ up to the fourth-order time derivatives as

$$
\begin{aligned}
& h=h_{0}+h_{1}+h_{2}+h_{3}+h_{4}, \\
& \widetilde{h}=\widetilde{h}_{0}+\widetilde{h}_{1}+\widetilde{h}_{2}+\widetilde{h}_{3}+\widetilde{h}_{4},
\end{aligned}
$$

respectively, where

$$
\begin{aligned}
& h_{0}=\int_{\mathbf{q}} G_{q} H_{\mathbf{q}} e^{\mathrm{iq} \cdot \mathbf{R}(\omega)}, \\
& h_{1}=h_{1,1}+h_{1,2} \\
& =\mathrm{i} \int_{\mathbf{q}}(\mathbf{v} \cdot \mathbf{q}) G_{q}^{2} H_{\mathbf{q}} e^{\mathrm{i} \mathbf{q} \cdot \mathbf{R}(\omega)}-\int_{\mathbf{q}} G_{q}^{2}\left(\frac{\partial H_{\mathbf{q}}}{\partial t}\right) e^{\mathrm{i} \mathbf{q} \cdot \mathbf{R}(\omega)}, \\
& h_{2}=h_{2,1}+h_{2,2}+h_{2,3} \\
& =-\mathrm{i} \int_{\mathbf{q}}(\dot{\mathbf{v}} \cdot \mathbf{q}) G_{q}^{3} H_{\mathbf{q}} e^{\mathrm{i} \mathbf{q} \cdot \mathbf{R}(\omega)} \\
& -\int_{\mathbf{q}}(\mathbf{v} \cdot \mathbf{q})^{2} G_{q}^{3} H_{\mathbf{q}} e^{\mathrm{iq} \cdot \mathbf{R}(\omega)} \\
& +\int_{\mathbf{q}} G_{q}^{3}\left(\frac{\partial^{2} H_{\mathbf{q}}}{\partial t^{2}}\right) e^{\mathrm{i} \mathbf{q} \cdot \mathbf{R}(\omega)}, \\
& h_{3}=-\mathrm{i} \int_{\mathbf{q}}(\mathbf{v} \cdot \mathbf{q})^{3} G_{q}^{4} H_{\mathbf{q}} e^{\mathrm{iq} \cdot \mathbf{R}(\omega)}, \\
& h_{4}=\int_{\mathbf{q}}(\mathbf{v} \cdot \mathbf{q})^{4} G_{q}^{5} H_{\mathbf{q}} e^{\mathrm{i} \mathbf{q} \cdot \mathbf{R}(\omega)}, \\
& \widetilde{h}_{0}=\mathrm{i} \int_{\mathbf{q}}(\mathbf{n} \cdot \mathbf{q}) G_{q} H_{\mathbf{q}} e^{\mathrm{i} \mathbf{q} \cdot \mathbf{R}(\omega)}, \\
& \widetilde{h}_{1}=\widetilde{h}_{1,1}+\widetilde{h}_{1,2} \\
& =-\int_{\mathbf{q}}(\mathbf{n} \cdot \mathbf{q})(\mathbf{v} \cdot \mathbf{q}) G_{q}^{2} H_{\mathbf{q}} e^{\mathrm{iq} \cdot \mathbf{R}(\omega)} \\
& -\mathrm{i} \int_{\mathbf{q}}(\mathbf{n} \cdot \mathbf{q}) G_{q}^{2}\left(\frac{\partial H_{\mathbf{q}}}{\partial t}\right) e^{\mathrm{i} \mathbf{q} \cdot \mathbf{R}(\omega)}, \\
& \widetilde{h}_{2}=\widetilde{h}_{2,1}+\widetilde{h}_{2,2}+\widetilde{h}_{2,3} \\
& =\int_{\mathbf{q}}(\mathbf{n} \cdot \mathbf{q})(\dot{\mathbf{v}} \cdot \mathbf{q}) G_{q}^{3} H_{\mathbf{q}} e^{\mathrm{i} \mathbf{q} \cdot \mathbf{R}(\omega)} \\
& -\mathrm{i} \int_{\mathbf{q}}(\mathbf{n} \cdot \mathbf{q})(\mathbf{v} \cdot \mathbf{q})^{2} G_{q}^{3} H_{\mathbf{q}} \mathrm{e}^{\mathrm{i} \mathbf{q} \cdot \mathbf{R}(\omega)} \\
& +\mathrm{i} \int_{\mathbf{q}}(\mathbf{n} \cdot \mathbf{q}) G_{q}^{3}\left(\frac{\partial^{2} H_{\mathbf{q}}}{\partial t^{2}}\right) e^{\mathrm{i} \mathbf{q} \cdot \mathbf{R}(\omega)}, \\
& \widetilde{h}_{3}=\int_{\mathbf{q}}(\mathbf{n} \cdot \mathbf{q})(\mathbf{v} \cdot \mathbf{q})^{3} G_{q}^{4} H_{\mathbf{q}} e^{\mathrm{i} \mathbf{q} \cdot \mathbf{R}(\omega)}, \\
& \widetilde{h}_{4}=\mathrm{i} \int_{\mathbf{q}}(\mathbf{n} \cdot \mathbf{q})(\mathbf{v} \cdot \mathbf{q})^{4} G_{q}^{5} H_{\mathbf{q}} e^{\mathrm{i} \mathbf{q} \cdot \mathbf{R}(\omega)}
\end{aligned}
$$




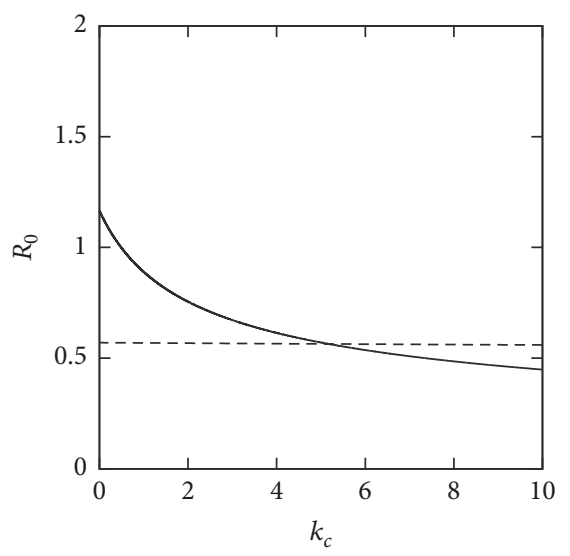

(a)

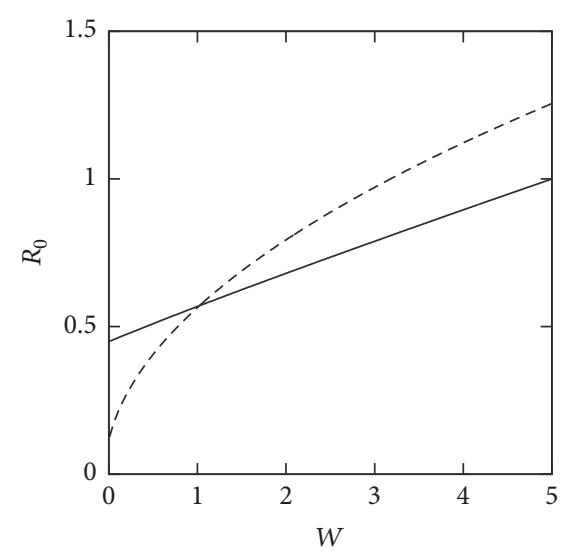

(b)

FIgURE 2: Dependence of $R_{0}$ on $k_{c}$ and $W$ in the stationary state. (a) Dependence of $R_{0}$ on $k_{c} . W=1.0$. The solid and dashed curves represent the cases of $\alpha=0$ and 5, respectively. (b) Dependence of $R_{0}$ on $W . k_{c}=5.0$. The solid and dashed curves represent the cases of $\alpha=0.1$ and 5.0 , respectively.

with

$$
G_{q}=\frac{1}{q^{2}+\beta} .
$$

In the above expansions, we omitted the term proportional to $(\mathbf{q} \cdot \mathbf{v})\left(\partial H_{\mathbf{q}} / \partial t\right)$ in the expressions of $h_{2}$ and $\widetilde{h}_{2}$. In the derivation of $h_{3}$ and $\widetilde{h}_{3}$, the terms proportional to $\left(\partial^{3} H_{\mathbf{q}} / \partial t^{3}\right),(\mathbf{q} \cdot \mathbf{v})\left(\partial^{2} H_{\mathbf{q}} / \partial t^{2}\right),(\mathbf{q} \cdot \mathbf{v})^{2}\left(\partial H_{\mathbf{q}} / \partial t\right),(\mathbf{q} \cdot \dot{\mathbf{v}})\left(\partial H_{\mathbf{q}} / \partial t\right)$, $(\mathbf{q} \cdot \ddot{\mathbf{v}}) H_{\mathbf{q}}$, and $(\mathbf{q} \cdot \mathbf{v})(\mathbf{q} \cdot \dot{\mathbf{v}}) H_{\mathbf{q}}$ were omitted. In the derivation of $h_{4}$ and $\widetilde{h}_{4}$, all the terms of the derivatives of $\mathbf{v}$ and $H_{\mathbf{q}}$ with respect to time were omitted. These terms were omitted, because they include higher order derivatives of time and are smaller than the remaining terms, or they vanish in the later integral $(1 / \Omega) \int d \omega \mathbf{R}(\omega)$. due to the orthogonality relation of trigonometric functions. We remark that we include up to the third-order of $\mathbf{v}: h_{3}$ and $\widetilde{h}_{3}$, for the derivation of the equation of motion of the spot. The justification for considering terms up to this order is discussed later. On the other hand, for the derivation of equations of motion of tensors, we include up to the fourth order of $\mathbf{v}: h_{4}$ and $\widetilde{h}_{4}$. The justification for considering terms up to this order is discussed in Section 4.4.

When the spot is in a stationary motionless state, we assume that the spot is a circle with radius $R_{0}$. The values of $v$ and $(\mathbf{n} \cdot \nabla) v$ at the interface are denoted as $h_{0}^{(0)}$ and $\widetilde{h}_{0}^{(0)}$, respectively. Using (34), $h_{0}^{(0)}$ is given by $h_{0}$ with the substitutions $H_{\mathbf{q}}=H_{\mathbf{q}}^{(0)}$ and $\mathbf{R}=\mathbf{R}^{(0)}$, where $\mathbf{R}^{(0)}=R_{0} \mathbf{e}_{r}$. Similarly, $\widetilde{h}_{0}^{(0)}$ is given using (35). When $\epsilon$ is small but finite, substituting $h=h_{0}^{(0)}$ and $\widetilde{h}=\widetilde{h}_{0}^{(0)}$ into $\tau C(h)$ with $V=0$, (14) becomes

$$
P\left(h_{0}^{(0)}\right)+Q\left(h_{0}^{(0)}\right) \widetilde{h}_{0}^{(0)}-\frac{\epsilon}{R_{0}}+L=0,
$$

where $\kappa=-1 / R_{0}$ is used. We note that the Lagrange multiplier $L$ in (37) can be absorbed into the constant term $p_{0}$ in $P\left(h_{0}^{(0)}\right)$. In the limit $\epsilon \rightarrow 0$, we can calculate $h_{0}^{(0)}$ and $\widetilde{h}_{0}^{(0)}$ by using $\bar{v}(r)$, which is given by (13) (the validation is given in Appendix B). Using these expressions, (37) gives the dependence of $R_{0}$ on $k_{c}$ and $W$. The numerical results obtained by using (13) and (17) are shown in Figure 2(a). When $\alpha=0, R_{0}$ monotonically decreases with $k_{c}$. However, for large $\alpha, R_{0}$ depends weakly on $k_{c}$ and it is almost constant because of the global coupling; large global coupling suppresses variations in the area of the spot resulting in constant $R_{0}$. The dependence of $R_{0}$ on $W$ when $k_{c}$ is large is shown in Figure 2(b). From (9), it can be seen that, for large $\alpha, R_{0}$ approximately satisfies $\pi R_{0}^{2} \sim W$, and, therefore, $R_{0}$ is proportional to the square root of $W$ (see the dashed curve in Figure 2(b)).

When the spot moves with an infinitesimal velocity, $h$ and $\widetilde{h}$ deviate from $h_{0}^{(0)}$ and $\widetilde{h}_{0}^{(0)}$, respectively. By putting small deviations as $\delta h=h-h_{0}^{(0)}$ and $\delta \widetilde{h}=\widetilde{h}-\widetilde{h}_{0}^{(0)}$, we iteratively derive $\delta h$ and $\delta \tilde{h}$ in a power series of $\tau C$ up to the third order. The procedure consists of two steps. We first expand $\tau C$ given by (17) in a power series of $\delta h$ and $\delta \tilde{h}$. Then, we solve the equation in terms of $\delta h$ and expand it by $\tau C$. When the stationary motionless spot is destabilized into a moving spot, $\tau C$ is expanded up to the second-order of $\delta h$ and $\delta \widetilde{h}$ as

$$
\begin{aligned}
& \tau C \\
& =\left(\frac{\partial P}{\partial h}+\frac{\partial Q}{\partial h} \widetilde{h}_{0}^{(0)}\right)_{0} \delta h+(Q)_{0} \delta \widetilde{h} \\
& \quad+\frac{1}{2}\left[\left(\frac{\partial^{2} P}{\partial h^{2}}+\frac{\partial^{2} Q_{h^{2}}(0)}{\partial h^{2}}\right)_{0} \delta h^{2}+2\left(\frac{\partial Q}{\partial h}\right)_{0} \delta h \delta \tilde{h}\right],
\end{aligned}
$$

where, for a function $A(h),(A)_{0}$ implies $A\left(h_{0}^{(0)}\right)$. Up to the first order of $\delta h$ and $\delta \tilde{h},(38)$ results in $\tau C=(\partial P / \partial h+$ $\left.\partial Q / \partial h \widetilde{h}_{0}^{(0)}\right)_{0} \delta h+(Q)_{0} \delta \tilde{h}$. For higher order corrections, a supplementary relation between $\delta h$ and $\delta \tilde{h}$ is necessary. In order to relate $\delta h$ with $\delta \tilde{h}$, we consider the profile of $v(\mathbf{r})$ in the radially symmetric function given by (13), where $v(\mathbf{r})$ is 
a function of $r(=|\mathbf{r}|)$ denoted by $\bar{v}(r)$. For this function, $(\mathbf{n} \cdot \nabla) \bar{v}=d \bar{v} / d r$. Then, $\bar{v}\left(R_{0}+\delta r\right)$ and $(\mathbf{n} \cdot \nabla) \bar{v}\left(R_{0}+\delta r\right)$ are expanded around $\bar{v}\left(R_{0}\right)$ and $d \bar{v}\left(R_{0}\right) / d r$ for small $\delta r$ as

$$
\begin{aligned}
\bar{v}\left(R_{0}+\delta r\right) & \sim \bar{v}\left(R_{0}\right)+\left(\frac{d \bar{v}}{d r}\right)_{r=R_{0}} \delta r, \\
(\mathbf{n} \cdot \nabla) \bar{v}\left(R_{0}+\delta r\right) & \sim \frac{d \bar{v}\left(R_{0}\right)}{d r}+\left(\frac{d^{2} \bar{v}}{d r^{2}}\right)_{r=R_{0}} \delta r,
\end{aligned}
$$

respectively. In (39) and (40), $(A(r))_{r=R_{0}}$ implies $A\left(R_{0}\right)$. We make an ansatz that $\delta h$ and $\delta \widetilde{h}$ are not independent but have a linear relation such that $\delta \widetilde{h}=k \delta h$ with $k=\left(\bar{v}^{\prime \prime} / \bar{v}^{\prime}\right)_{r=R_{0}}$, where the prime corresponds to the derivative with respect to $r$. This assumption enables us to calculate $\delta h$ as a function of $\tau C$. Substituting $\delta \tilde{h}=k \delta h$ into (38), $\tau C$ becomes a quadratic equation of $\delta h$ as

$$
\tau C=(a+k b) \delta h+m_{1} \delta h^{2},
$$

where

$$
\begin{aligned}
a & =\left(\frac{\partial P}{\partial h}+\frac{\partial Q}{\partial h} \widetilde{h}_{0}^{(0)}\right)_{0}, \\
b & =(Q)_{0}, \\
m_{1} & =\frac{1}{2}\left[\left(\frac{\partial^{2} P}{\partial h^{2}}+\frac{\partial^{2} Q}{\partial h^{2}} \widetilde{h}_{0}^{(0)}\right)_{0}+2 k\left(\frac{\partial Q}{\partial h}\right)_{0}\right] .
\end{aligned}
$$

As a result of numerical calculations, we remark that $b>0$ and $a<0$ for small $h_{0}^{(0)}$, but $a>0$ for large $h_{0}^{(0)}$. By solving (41) in terms of $\delta h$ for small $\tau C$, the solution is expanded up to the second order of $\tau C$ as

$$
\delta h=\left(\frac{\tau C}{a+k b}\right)-\frac{m_{1}}{a+k b}\left(\frac{\tau C}{a+k b}\right)^{2} .
$$

Here, it should be noted that although there are two solutions for (41), we chose one solution such that $\delta h \rightarrow \tau C /(a+k b)$ when the second-order term $(\tau C)^{2}$ is neglected.

In order to obtain $\delta h$ up to the third order of $\tau C, \tau C$ given by (17) is expanded up to the third order of $\delta h$ and $\delta \tilde{h}$. Using $\delta \widetilde{h}=k \delta h, \tau C$ becomes a cubic equation of $\delta h$ as

$$
\tau C=(a+k b) \delta h+m_{1} \delta h^{2}+m_{2} \delta h^{3},
$$

where $m_{2}$ is given by

$$
m_{2}=\frac{1}{6}\left[\left(\frac{\partial^{3} P}{\partial h^{3}}+\frac{\partial^{3} Q^{3}}{\partial h^{3}} \widetilde{h}_{0}^{(0)}\right)_{0}+3 k\left(\frac{\partial^{2} Q}{\partial h^{2}}\right)_{0}\right] .
$$

In order to obtain the third-order term of $\tau C$ in $\delta h$, we add a correction $\Delta\left(\sim O(\tau C)^{3}\right)$ to $\delta h$ given by (43) and substitute it into (44). On solving this equation in terms of $\Delta, \delta h$ up to the third order of $\tau C$ is obtained as

$$
\begin{aligned}
\delta h= & \left(\frac{\tau C}{a+k b}\right)-\frac{m_{1}}{a+k b}\left(\frac{\tau C}{a+k b}\right)^{2} \\
& +\frac{1}{a+k b}\left[\frac{2 m_{1}^{2}}{a+k b}-m_{2}\right]\left(\frac{\tau C}{a+k b}\right)^{3} .
\end{aligned}
$$

We remark that (46) is obtained by another method; by applying Cardano's formula to (44) and expanding the solution up to the third order of $\tau C$, we obtain the same result as that of (46).

In the above process, we iteratively derived $\delta h$ up to the third order of $\tau C$. We replace $k \delta h \sim \delta \widetilde{h}$ in (46), and finally the power series expansion of $a \delta h+b \delta \tilde{h}$ in terms of $\tau C$ is obtained as

$$
a \delta h+b \delta \tilde{h}=M_{1}(\tau C)+M_{2}(\tau C)^{2}+M_{3}(\tau C)^{3},
$$

where $M_{i}(i=1,2,3)$ is defined by

$$
\begin{aligned}
& M_{1}=1, \\
& M_{2}=-\frac{m_{1}}{(a+b k)^{2}}, \\
& M_{3}=\frac{1}{(a+b k)^{3}}\left[\frac{2 m_{1}^{2}}{a+b k}-m_{2}\right] .
\end{aligned}
$$

In the absence of chemotaxis $(Q=0),(47)$ reproduces the result that was obtained in [16].

Using (14) and (47), we derive the equation of motion of the spot by operating both sides of $(47)$ with $(1 / \Omega) \int d \omega \mathbf{R}(\omega)$. For the left hand side, we put

$$
\begin{gathered}
\frac{1}{\Omega} \int d \omega \mathbf{R}(\omega) \delta h=\delta \mathbf{h}=\mathbf{h}_{1}+\mathbf{h}_{2}+\mathbf{h}_{3}, \\
\frac{1}{\Omega} \int d \omega \mathbf{R}(\omega) \delta \tilde{h}=\delta \tilde{\mathbf{h}}=\tilde{\mathbf{h}}_{1}+\widetilde{\mathbf{h}}_{2}+\tilde{\mathbf{h}}_{3},
\end{gathered}
$$

where $\mathbf{h}_{4}$ and $\widetilde{\mathbf{h}}_{4}$ are neglected because these terms are higher than or equal to $O\left(\mathbf{v}^{4}\right)$. For the calculation of the right hand side of (47), we use (14). The magnitudes of $\epsilon \kappa$ and $L$ were discussed in [16]; $L$ is independent of $\phi_{r}$, and, owing to the periodicity of the function, $\int d \omega \mathbf{R}(\omega) L=\int d \omega \mathbf{R}(\omega) L^{3}=$ $\int d \omega \mathbf{R}(\omega) V^{2} L=0$. In addition, $L \sim O\left(\mathbf{v}^{2}\right)$, and, therefore, $\int d \omega \mathbf{R}(\omega) V L^{3}$ can be neglected up to the third order of $\mathbf{v}$. As the translational motion of the spot is incorporated in $\boldsymbol{\rho}$, we chose $c_{ \pm 1}=0$ in the expansion of $\delta R\left(\phi_{r}\right)$. This results in $\epsilon \int d \omega \mathbf{R}(\omega) \kappa=0$ up to the first order of the deformation. The terms $\epsilon \int d \omega \mathbf{R}(\omega) \kappa V L^{2}$ and $\epsilon \int d \omega \mathbf{R}(\omega) \kappa L^{2}$, and the higher order terms of $\epsilon$ were small; therefore, they were neglected. Following the above discussion, we neglect the term $(\epsilon \kappa+L)$ in (14).

By carrying the integral over $\phi_{r}$, the following equation is obtained:

$$
a \delta \mathbf{h}+b \delta \tilde{\mathbf{h}}=\tau \mathbf{v}+\frac{3}{4} M_{3} \tau^{3} \mathbf{v}|\mathbf{v}|^{2}
$$

where

$$
\begin{aligned}
& \delta \mathbf{h}=\mathbf{h}_{1,1}+\mathbf{h}_{2,1}+\mathbf{h}_{3}, \\
& \delta \widetilde{\mathbf{h}}=\widetilde{\mathbf{h}}_{1,1}+\widetilde{\mathbf{h}}_{2,1}+\widetilde{\mathbf{h}}_{3} .
\end{aligned}
$$

Here, $\mathbf{h}_{1,1}=(1 / \Omega) \int d \omega \mathbf{R}(\omega) h_{1,1}$. The other terms on the right hand side of (51) are defined similarly. In the expansion 


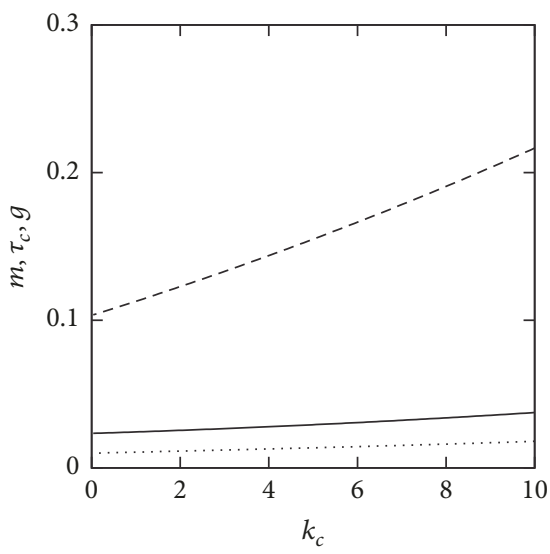

(a)

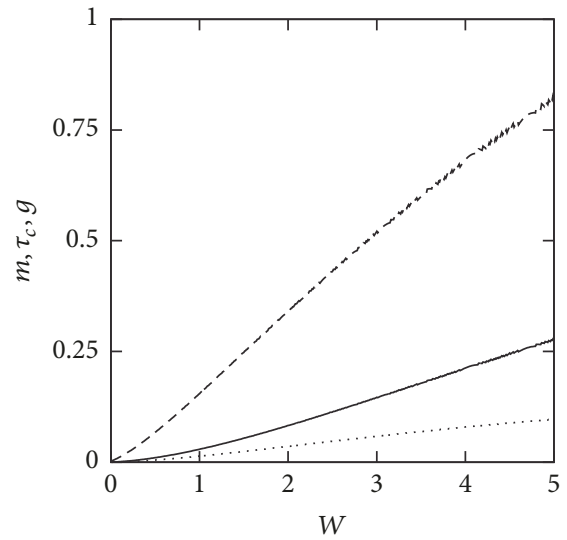

(b)

FIGURE 3: Dependence of $m, \tau_{c}$, and $g$ on $k_{c}$ and $W$. The solid, dashed, and dotted curves represent $m, \tau_{c}$, and $g$, respectively. (a) Dependence of $m, \tau_{c}$, and $g$ on $k_{c} . \alpha=5.0$ and $W=1.0$. In the calculation of $g, \tau$ is chosen as $\tau=\tau_{c}$. As $M_{3}$ is positive, $g$ for the case $\tau<(>) \tau_{c}$ is smaller (larger) than this curve. (b) Dependence of $m, \tau_{c}$, and $g$ on $W . \alpha=5.0$ and $k_{c}=5.0$. In the calculation of $g, \tau$ is chosen as $\tau=\tau_{c}$.

(51), we neglected the terms $\mathbf{h}_{1,2}$ and $\mathbf{h}_{2,3}$, because these terms yielded only the terms $\dot{c}_{ \pm 1}$ and $\ddot{c}_{ \pm 1}$, respectively; however, these terms are set to zero. $\mathbf{h}_{2,2}$ disappeared in the integral over $\phi_{r}$ owing to the orthogonality relation of trigonometric functions [15]. Owing to the same reasons, the terms $\widetilde{\mathbf{h}}_{1,2}$, $\widetilde{\mathbf{h}}_{2,3}$, and $\widetilde{\mathbf{h}}_{2,2}$ did not exist. In the integral over $\phi_{r}$, there is no contribution from the second-order term of $\tau C$ owing to the orthogonality relation of trigonometric function. This supports the justification to include terms up to the third order of $\tau C$ in (47) for the minimal equation of motion of a spot. For the balance of order, it is sufficient to include terms up to the third order of $\mathbf{v}$ in $\delta \mathbf{h}$ and $\delta \tilde{\mathbf{h}}$.

After practical calculations of $\delta \mathbf{h}$ and $\delta \widetilde{\mathbf{h}}$, we finally obtain the equation of motion of the spot as

$$
m \dot{\mathbf{v}}+\left(\tau-\tau_{c}\right) \mathbf{v}+g \mathbf{v}|\mathbf{v}|^{2}=a \delta \mathbf{h}_{1,1}^{(0)}+b \delta \widetilde{\mathbf{h}}_{1,1}^{(0)},
$$

where $\delta \mathbf{h}_{1,1}^{(0)}$ is defined as $\delta \mathbf{h}_{1,1}^{(0)}=\mathbf{h}_{1,1}-\mathbf{h}_{1,1}^{(0)}$. Here, $\mathbf{h}_{1,1}^{(0)}$ is given by $\mathbf{h}_{1,1}$ with the substitutions $H_{\mathbf{q}}=H_{\mathbf{q}}^{(0)}$ and $\mathbf{R}=\mathbf{R}^{(0)} . \delta \widetilde{\mathbf{h}}_{1,1}^{(0)}$ is similarly defined with $\delta \mathbf{h}_{1,1}^{(0)}$. The coefficients $m, \tau_{c}$, and $g$ on the left hand side of (52) are given by

$$
\begin{aligned}
m= & -a R_{0} F(1,3,1,1)-b R_{0} F_{d}(2,3,1,1), \\
\tau_{c}= & -a R_{0} F(1,2,1,1)-b R_{0} F_{d}(2,2,1,1), \\
g= & \frac{3}{4} M_{3} \tau^{3}-\frac{3}{4} a R_{0} F(3,4,1,1) \\
& -\frac{3}{4} b R_{0} F_{d}(4,4,1,1),
\end{aligned}
$$

respectively, where we defined $F(i, j, k, l)$ and $F_{d}(i, j, k, l)$ as

$$
\begin{aligned}
F(i, j, k, l) & =\int_{0}^{\infty} d q q^{i} G_{q}^{j} J_{k} J_{l}, \\
F_{d}(i, j, k, l) & =\int_{0}^{\infty} d q q^{i} G_{q}^{j} J_{k} \frac{\partial}{\partial\left(q R_{0}\right)} J_{l},
\end{aligned}
$$

respectively, where $i, j, k$, and $l$ are integers and $J_{n}\left(q R_{0}\right)$ is written as $J_{n}$ for brevity.

Equation (52) has the same form as the one in the absence of chemotaxis [16]. It is a Newtonian equation of the spot; the effective mass $m$, damping coefficient $\tau_{c}$, intensity of the cubic nonlinear term $g$, and the right hand side of (52) form the coupling term between deformation and velocity in the following subsection. When $\left(\tau-\tau_{c}\right)>0$, the motionless spot is stable; however, the motionless spot is destabilized into a moving spot for $\left(\tau-\tau_{c}\right)<0$. When $g$ is positive, the third-order nonlinear coupling term $g \mathbf{v}|\mathbf{v}|^{2}$ suppresses the divergence for large $|\mathbf{v}|$. It is necessary that $m, \tau_{c}$, and $g$ are positive for the existence of a stable moving spot. The dependence of $m, \tau_{c}$, and $g$ on $k_{c}$ and $W$ is shown in Figure 3 . We see that these parameters are positive and monotonically increase with $k_{c}$ and $W$.

In the derivation of (47), we made an ansatz for the relation between $\delta h$ and $\delta \widetilde{h}$ such that $\delta \widetilde{h}=k \delta h$ with $k=$ $\left(\bar{v}^{\prime \prime} / \bar{v}^{\prime}\right)_{r=R_{0}}$. Equation (55) suggests that $g$ depends explicitly on $k$ through $M_{3}$. Through preliminary research, it is found that if $k$ is chosen as a positive constant, the property that $g$ is a positive and monotonically increasing function of $k_{c}$ and $W$ holds. From the above, it is seen that $m, \tau_{c}$, and $g$ are positive for any value of $k_{c}$ and $W$, and, therefore, we fix $W$ and examine the motion of the spot in the range $k_{c} \geq 0$.

4.3. Definitions of Tensors. In order to express the deformation of the spot, we introduce tensors. We rewrite the vector form (52) of each component, and, by denoting $\mathbf{v}=\left(v_{1}, v_{2}\right)$, the $\alpha$ component $(\alpha=1,2)$ of (52) becomes

$$
m \dot{v}_{\alpha}+\left(\tau-\tau_{c}\right) v_{\alpha}+g v_{\alpha}|\mathbf{v}|^{2}=a \delta h_{1,1(\alpha)}^{(0)}+b \delta \widetilde{h}_{1,1(\alpha)}^{(0)},
$$

where $\delta h_{1,1(\alpha)}^{(0)}$ and $\delta \widetilde{h}_{1,1(\alpha)}^{(0)}$ correspond to the $\alpha$ component of $\delta \mathbf{h}_{1,1}^{(0)}$ and $\delta \widetilde{\mathbf{h}}_{1,1}^{(0)}$, respectively. Using the tensors, the right hand side of (58) is expressed in a simple form. The second rank tensor $\mathbf{S}$ and the third rank tensor $\mathbf{U}$ represent the elliptical deformation and the head-tail asymmetric deformation 
( $2 \pi / 3$-periodic deformation in the radial direction) of the spot, respectively. These tensors are traceless and symmetric. The detailed definitions are given in [16]. The deformation $\delta R\left(\phi_{r}\right)$ is expanded using the coefficients $c_{n}$ as given by (25); $c_{ \pm n}$ corresponds to a $(2 \pi / n)$-periodic deformation.

We first introduce a second-rank tensor $S_{\alpha \beta}(\alpha, \beta=1,2)$ using $c_{ \pm 2}$ as follows:

$$
\begin{aligned}
& S_{11}=-S_{22}=c_{2}+c_{-2}=\frac{\delta_{2}}{2} \cos 2 \phi_{2}, \\
& S_{12}=S_{21}=\mathrm{i}\left(c_{2}-c_{-2}\right)=\frac{\delta_{2}}{2} \sin 2 \phi_{2},
\end{aligned}
$$

where $\delta_{2}$ is a positive constant, which represents the radial deviation of the spot from $R_{0}$, and $\phi_{2}$ is the angle between the long axis of the ellipse and the $x$-axis. The tensor elements (59) are represented by using a normal vector $\mathbf{N}=$ $\left(\cos \phi_{2}, \sin \phi_{2}\right)$ along the long axis as

$$
S_{\alpha \beta}=\delta_{2}\left(N_{\alpha} N_{\beta}-\frac{\delta_{\alpha \beta}}{2} \mathbf{N}^{2}\right) .
$$

The tensor $\mathbf{S}$ is the same as the nematic order parameter tensor in liquid crystals [34]. For an elliptical spot, $R\left(\phi_{r}\right)$ is represented as

$$
R\left(\phi_{r}\right)=R_{0}+\frac{\delta_{2}}{2} \cos 2\left(\phi_{r}-\phi_{2}\right) .
$$

Next, in order to describe the head-tail asymmetric deformation, we first define $T_{1}$ and $T_{2}$ using $c_{ \pm 3}$ as

$$
\begin{aligned}
& T_{1}=c_{3}+c_{-3}=\delta_{3} \cos 3 \phi_{3}, \\
& T_{2}=\mathrm{i}\left(c_{3}-c_{-3}\right)=\delta_{3} \sin 3 \phi_{3},
\end{aligned}
$$

respectively, where $\delta_{3}$ is a positive constant and $\phi_{3}$ is the angle between one of the long axes of the deformed spot and the $x$ axis. In order to relate $T_{1}$ and $T_{2}$ with a tensor, we introduce the third-rank tensor $U_{\alpha \beta \gamma}(\alpha, \beta$, and $\gamma=1$ or 2$)$. The tensor elements $U_{\alpha \beta \gamma}$ are represented by using vectors $\mathbf{N}^{(m)}(m=$ 1,2 , and 3 ) as

$$
U_{\alpha \beta \gamma}=\frac{4 \delta_{3}}{3} \sum_{m} N_{\alpha}^{(m)} N_{\beta}^{(m)} N_{\gamma}^{(m)},
$$

where the normal vectors $\mathbf{N}^{(m)}$ are defined by

$$
\begin{aligned}
& \mathbf{N}^{(1)}=\left(\cos \phi_{3}, \sin \phi_{3}\right), \\
& \mathbf{N}^{(2)}=\left(\cos \left(\phi_{3}+\frac{2 \pi}{3}\right), \sin \left(\phi_{3}+\frac{2 \pi}{3}\right)\right), \\
& \mathbf{N}^{(3)}=\left(\cos \left(\phi_{3}-\frac{2 \pi}{3}\right), \sin \left(\phi_{3}-\frac{2 \pi}{3}\right)\right) .
\end{aligned}
$$

The tensor $\mathbf{U}$ is the same as the order parameter for banana (tetrahedral nematic) liquid crystals in two dimensions [35, 36]. We obtain the relations between the tensor elements $U_{\alpha \beta \gamma}$ and $T_{1}$ and $T_{2}$ as $U_{111}=T_{1}, U_{222}=-T_{2}$, and

$$
\begin{aligned}
& U_{111}=-U_{122}=-U_{212}=-U_{221}, \\
& U_{222}=-U_{112}=-U_{121}=-U_{211} .
\end{aligned}
$$

The spot with head-tail asymmetric deformation is represented as

$$
R\left(\phi_{r}\right)=R_{0}+\delta_{3} \cos 3\left(\phi_{r}-\phi_{3}\right)
$$

The terms $\delta h_{1,1(\alpha)}^{(0)}$ and $\delta \widetilde{h}_{1,1(\alpha)}^{(0)}$ in (58) are expressed using tensors. The detailed calculations are given in Appendix C. The final form is as follows:

$$
\begin{aligned}
& \delta h_{1,1(\alpha)}^{(0)}=-a^{*} \sum_{\beta} S_{\alpha \beta} v_{\beta}, \\
& \delta \widetilde{h}_{1,1(\alpha)}^{(0)}=-\widetilde{a}^{*} \sum_{\beta} S_{\alpha \beta} v_{\beta},
\end{aligned}
$$

where

$$
\begin{aligned}
a^{*}= & a_{1}^{*}+a_{2}^{*}, \\
a_{1}^{*}= & -\frac{R_{0}}{2} F(2,2,1,2), \\
a_{2}^{*}= & -\frac{1}{2}\left[R_{0} F(2,2,1,2)-3 F(1,2,1,1)\right], \\
\tilde{a}^{*}= & \tilde{a}_{1}^{*}+\tilde{a}_{2}^{*}, \\
\tilde{a}_{1}^{*}= & -\frac{R_{0}}{8}[-F(3,2,1,2)+3 F(3,2,1,1)] \\
& -\frac{3}{4} F(2,2,0,1), \\
\tilde{a}_{2}^{*}= & -2\left[\frac{R_{0}}{4} F_{d}(2,2,2,1)-F_{d}(1,2,1,1)\right] .
\end{aligned}
$$

Thus, (58) is written using $S_{\alpha \beta}$ in the form

$$
m \dot{v}_{\alpha}+\left(\tau-\tau_{c}\right) v_{\alpha}+g v_{\alpha}|\mathbf{v}|^{2}=-\bar{a} \sum_{\beta} S_{\alpha \beta} v_{\beta}
$$

where $\bar{a}=\left(a a^{*}+b \widetilde{a}^{*}\right) \cdot \delta h_{1,1(\alpha)}^{(0)}$ and $\delta \widetilde{h}_{1,1(\alpha)}^{(0)}$ yield the $\mathbf{S v}$ term. This is due to the periodicity of the function in the integral over $\phi_{r}$; in the expansion of $\delta \mathbf{R}\left(\phi_{r}\right)$ and $H_{\mathbf{q}}^{(1)}\left(\phi_{r}\right)$, only $c_{ \pm 2}$ terms contribute to the nonzero integral, resulting in the $\mathbf{S v}$ term. Equation (73) suggests that the motionless spot is destabilized into a moving spot for $\tau_{c}>\tau$, and the velocity of the spot is approximately given by $|\mathbf{v}|^{2} \sim\left(\tau_{c}-\tau\right) / g$. The deformation and velocity coupling term $\mathbf{S v}$ modifies the straight motion of the spot.

4.4. Time Evolution Equations of Tensors. In the previous subsection, we defined the tensors and described the equation of motion of the spot by using tensors, including the timedependent tensor $S_{\alpha \beta}$. In this subsection, we derive the time evolution equations of the tensors up to $\sim O\left(\mathbf{v}^{4}\right)$. We first discuss the order of $\mathbf{v}, S_{\alpha \beta}$, and $U_{\alpha \beta \gamma}$, following [16]. From (73), the motionless spot is critical at $\tau=\tau_{c}$, and the moving spot occurs supercritically with increasing $\left(\tau_{c}-\tau\right)$. We put $\delta=\tau_{c}-\tau$ for the smallness parameter. The time is scaled by $\widehat{t}=t \delta$, an all the terms in (73) are of the order $O\left(\delta^{3 / 2}\right)$. 
Here $\mathbf{v} \sim O\left(\delta^{1 / 2}\right)$ and $\mathbf{S} \sim O(\delta)$; therefore $S_{\alpha \beta} \sim O\left(\mathbf{v}^{2}\right)$. In the later calculations, we can confirm that $S_{\alpha \beta} \sim O\left(\mathbf{v}^{2}\right)$ and $U_{\alpha \beta \gamma} \sim O\left(\mathbf{v}^{3}\right)$. In the derivation of (73), we omitted the terms that include higher order derivatives of time. We estimate these terms, for example, $d^{2} \mathbf{v} / d t^{2} \sim O\left(\delta^{5 / 2}\right), \mathbf{S S} \mathbf{v} \sim O\left(\delta^{5 / 2}\right)$, $(\mathbf{q} \cdot \mathbf{v}) \partial H_{\mathbf{q}} / \partial t \sim O\left(\delta^{5 / 2}\right), \partial^{3} H_{\mathbf{q}} / \partial t^{3} \sim O\left(\delta^{4}\right),(\mathbf{q} \cdot \mathbf{v}) \partial^{2} H_{\mathbf{q}} / \partial t^{2} \sim$ $O\left(\delta^{7 / 2}\right),(\mathbf{q} \cdot \mathbf{v})^{2} \partial H_{\mathbf{q}} / \partial t \sim O\left(\delta^{3}\right)$, and $(\mathbf{q} \cdot \dot{\mathbf{v}}) \partial H_{\mathbf{q}} / \partial t \sim O\left(\delta^{7 / 2}\right)$ in the expansion of $h$ and $\tilde{h}$. From these estimates, the omission of those terms is justified.

$\mathbf{S}$ and $\mathbf{U}$ are composed of $c_{ \pm 2}$ and $c_{ \pm 3}$, respectively, so that $c_{ \pm 2} \sim O\left(\mathbf{v}^{2}\right)$ and $c_{ \pm 3} \sim O\left(\mathbf{v}^{3}\right)$. The coupling terms such as $c_{n} c_{m}(|n|,|m| \geq 2)$ are much smaller. Therefore, we linearize (14) in terms of the deformation $\delta R$. Then, the time evolution equation of $\delta R$ becomes

$$
\tau \frac{\partial \delta R}{\partial t}=\frac{\epsilon}{R_{0}^{2}}\left(\frac{\partial^{2} \delta R}{\partial \phi_{r}^{2}}+\delta R\right)+a \delta h+b \delta \tilde{h}+L .
$$

We consider terms up to the fourth order of $\mathbf{v}$, where $\delta h$ and $\delta \widetilde{h}$ are given by

$$
\begin{aligned}
& \delta h=h-h_{0}^{(0)}=\delta h_{0}+\delta h_{1}+\delta h_{2}+\delta h_{3}+\delta h_{4}, \\
& \delta \widetilde{h}=\widetilde{h}-\widetilde{h}_{0}^{(0)}=\delta \widetilde{h}_{0}+\delta \widetilde{h}_{1}+\delta \widetilde{h}_{2}+\delta \widetilde{h}_{3}+\delta \widetilde{h}_{4},
\end{aligned}
$$

respectively. Here, $\delta h_{0}$ and $\delta h_{l}(l=1,2, \ldots, 4)$ are defined by (34) as $\delta h_{0}=h_{0}-h_{0}^{(0)}$ and $\delta h_{l}=h_{l}$, respectively. $\delta \widetilde{h}_{l}$ is similarly defined by (35). We expand $\delta h_{1}, \delta h_{2}, \delta \widetilde{h}_{1}$, and $\delta \widetilde{h}_{2}$ as $\delta h_{1}=\delta h_{1,1}+\delta h_{1,2}, \delta h_{2}=\delta h_{2,2}, \delta \widetilde{h}_{1}=\delta \tilde{h}_{1,1}+\delta \tilde{h}_{1,2}$, and $\delta \widetilde{h}_{2}=\delta \widetilde{h}_{2,2}$, respectively.

In order to derive the equation of motion of tensors, we calculate the first-order time derivative of $c_{n}$. The linear combination of $\dot{c}_{n}$ results in the equation of motion of tensors. In this process, $\dot{\mathbf{v}}$ terms in $\delta h_{2,1}$ and $\delta \widetilde{h}_{2,1}$ are neglected because these terms cause small order terms; $\sum_{\gamma} U_{\alpha \beta \gamma} \dot{v}_{\gamma}$ and $S_{\alpha \beta} \dot{v}_{\gamma}$ appear in the time evolution equation of $S_{\alpha \beta}$ and $U_{\alpha \beta \gamma}$, respectively. These terms are $\sum_{\gamma} U_{\alpha \beta \gamma} \dot{v}_{\gamma} \sim O\left(\mathbf{v}^{6}\right)$ and $S_{\alpha \beta} \dot{v}_{\gamma} \sim$ $O\left(\mathbf{v}^{5}\right)$ so that they do not contribute to the equation of motion of tensors. In addition, $\left(\partial^{2} H_{\mathbf{q}} / \partial t^{2}\right)$ terms in $\delta h_{2,3}$ and $\delta \widetilde{h}_{2,3}$ are neglected because these terms result in the second-order time derivative of $c_{n}$; these terms are $\ddot{c}_{ \pm 2} \sim O\left(\mathbf{v}^{6}\right)$ and $\ddot{c}_{ \pm 3} \sim O\left(\mathbf{v}^{7}\right)$ and they are small enough to be neglected.

We first derive the equation of motion of $S_{\alpha \beta}$. Using (74), we can obtain the time evolution equation of $c_{ \pm 2}$, and we derive the time evolution equation of $S_{\alpha \beta}$. The detailed derivation is given in Appendices $\mathrm{D}$ and $\mathrm{E}$. The time evolution equation of $S_{\alpha \beta}$ up to $O\left(\mathbf{v}^{4}\right)$ is

$$
\begin{aligned}
\Gamma_{2} \frac{d S_{\alpha \beta}}{d t}= & -K_{2} S_{\alpha \beta}+\bar{b}\left[v_{\alpha} v_{\beta}-\frac{\delta_{\alpha \beta}}{2}|\mathbf{v}|^{2}\right] \\
& +\bar{b}_{s}\left[v_{\alpha} v_{\beta}-\frac{\delta_{\alpha \beta}}{2}|\mathbf{v}|^{2}\right]|\mathbf{v}|^{2}+\bar{b}_{1} \sum_{\gamma} U_{\alpha \beta \gamma} v_{\gamma} \\
& +\bar{c}|\mathbf{v}|^{2} S_{\alpha \beta},
\end{aligned}
$$

where $\Gamma_{2}=\tau+a E_{2}+b \widetilde{E}_{2}, K_{2}=3 \epsilon / R_{0}^{2}+a D_{2}+b \widetilde{D}_{2}, \bar{b}=$ $a G_{1}+b \widetilde{G}_{1}, \bar{b}_{s}=-a G_{1}^{s}-b \widetilde{G}_{1}^{s}, \bar{b}_{1}=-a B_{3} / 2+b\left(3 \widetilde{A}^{*}-\widetilde{B}_{3}\right) / 2$, and $\bar{c}=-a\left(2 T_{2,2}-V_{2}\right)-b\left(2 \widetilde{T}_{2,2}-\widetilde{V}_{2}\right)$. All the parameters in these expressions are given in Appendix E. The second term in (77) originates from $\delta h_{2}$ and $\delta \widetilde{h}_{2}$, the third term from $\delta h_{4}$ and $\delta \widetilde{h}_{4}$, the fourth term from $\delta h_{1}$ and $\delta \widetilde{h}_{1}$, and the fifth term from $\delta h_{2}$ and $\delta \widetilde{h}_{2}$. Thus, in order to consider the terms up to $O\left(\mathbf{v}^{4}\right), \delta h_{4}$ and $\delta \widetilde{h}_{4}$ are necessary in the expansion equations (75) and (76). From the first and second terms in (77), we note that $S_{\alpha \beta} \sim O\left(\mathbf{v}^{2}\right)$.

Next, we consider the time evolution equation of $U_{\alpha \beta \gamma}$. Following procedures similar to those for $S_{\alpha \beta}$, the time evolution equation of $U_{\alpha \beta \gamma}$ up to $O\left(\mathbf{v}^{4}\right)$ is

$$
\begin{gathered}
\Gamma_{3} \frac{d U_{\alpha \beta \gamma}}{d t}=-K_{3} U_{\alpha \beta \gamma}+4 d_{1}\left[v_{\alpha} v_{\beta} v_{\gamma}\right. \\
\left.-\frac{|\mathbf{v}|^{2}}{4}\left(\delta_{\alpha \beta} v_{\gamma}+\delta_{\beta \gamma} v_{\alpha}+\delta_{\gamma \alpha} v_{\beta}\right)\right] \\
+\frac{2 d_{2}}{3}\left[S_{\alpha \beta} v_{\gamma}+S_{\beta \gamma} v_{\alpha}+S_{\gamma \alpha} v_{\beta}\right. \\
\left.-\sum_{\eta} \frac{v_{\eta}}{2}\left(\delta_{\alpha \beta} S_{\gamma \eta}+\delta_{\beta \gamma} S_{\alpha \eta}+\delta_{\gamma \alpha} S_{\beta \eta}\right)\right],
\end{gathered}
$$

where $\Gamma_{3}=\tau+a E_{3}+b \widetilde{E}_{3}$ and $K_{3}=8 \epsilon / R_{0}^{2}+a D_{3}+b \widetilde{D}_{3} \cdot d_{1}$ and $d_{2}$ are defined as

$$
\begin{aligned}
d_{1} & =-\frac{R_{0}}{4}\left[a F(3,4,1,3)+b F_{d}(4,4,1,3)\right], \\
d_{2} & =-a \frac{R_{0}}{2}\left[F(2,2,2,3)+F_{d}(2,2,1,1)\right] \\
- & b \frac{R_{0}}{2}\left[F_{d}(3,2,2,3)\right. \\
& \left.+\frac{1}{4}(F(3,2,1,3)-3 F(3,2,1,1))\right] \\
- & \frac{b}{2}[F(2,2,0,1)+F(2,2,1,2)],
\end{aligned}
$$

respectively. In the above equation, all the terms are $\sim O\left(\mathbf{v}^{3}\right)$. The terms of $O\left(\mathbf{v}^{4}\right)$ disappeared because of the orthogonality relation of trigonometric functions. The next higher order term is $O\left(\mathbf{v}^{5}\right)$, which is not included in expansions $\delta h$ and $\delta \widetilde{h}$ in (75) and (76). The second term in (78) originates from $\delta h_{3}$ and $\delta \widetilde{h}_{3}$, and the third term from $\delta h_{1}$ and $\delta \widetilde{h}_{1}$. From the first and second terms in (78), we note that $U_{\alpha \beta \gamma} \sim O\left(\mathbf{v}^{3}\right)$.

\section{Tensor Model}

Using (73), (77), and (78), we discuss the stationary solution, the stability conditions of straight motion, and the critical 
velocity of circular motion. For the sake of convenience, we rescale (73), (77), and (78) into

$$
\begin{aligned}
& \frac{d v_{\alpha}}{d t}=\gamma v_{\alpha}-v_{\alpha}|\mathbf{v}|^{2}-\bar{a}^{\prime} \sum_{\beta} S_{\alpha \beta} v_{\beta}, \\
& \frac{d S_{\alpha \beta}}{d t}=-\kappa S_{\alpha \beta}+\bar{b}^{\prime}\left[v_{\alpha} v_{\beta}-\frac{1}{2}|\mathbf{v}|^{2} \delta_{\alpha \beta}\right] \\
& +\bar{b}_{s}^{\prime}\left[v_{\alpha} v_{\beta}-\frac{1}{2}|\mathbf{v}|^{2} \delta_{\alpha \beta}\right]|\mathbf{v}|^{2} \\
& +\bar{b}_{1}^{\prime} \sum_{\gamma} U_{\alpha \beta \gamma} v_{\gamma}+\bar{c}^{\prime}|\mathbf{v}|^{2} S_{\alpha \beta}, \\
& \frac{d U_{\alpha \beta \gamma}}{d t}=-K U_{\alpha \beta \gamma} \\
& +d_{1}^{\prime}\left[v_{\alpha} v_{\beta} v_{\gamma}-\frac{|\mathbf{v}|^{2}}{4}\left(\delta_{\alpha \beta} v_{\gamma}+\delta_{\beta \gamma} v_{\alpha}+\delta_{\gamma \alpha} v_{\beta}\right)\right] \\
& +\frac{d_{2}^{\prime}}{3}\left[S_{\alpha \beta} v_{\gamma}+S_{\beta \gamma} v_{\alpha}+S_{\gamma \alpha} v_{\beta}\right. \\
& \left.-\sum_{\eta} \frac{v_{\eta}}{2}\left(\delta_{\alpha \beta} S_{\gamma \eta}+\delta_{\beta \gamma} S_{\alpha \eta}+\delta_{\gamma \alpha} S_{\beta \eta}\right)\right],
\end{aligned}
$$

respectively. We name (81)-(83) as the full tensor model [37]. In the above full tensor model, we chose parameters such that $\mathrm{g} / \mathrm{m}=1$ and put $\gamma=\left(\tau_{c}-\tau\right) / m$ and $\bar{a}^{\prime}=\bar{a} / m$ in (81). The other rescaled parameters in (82) and (83) were $\kappa=K_{2} / \Gamma_{2}$, $\bar{b}^{\prime}=\bar{b} / \Gamma_{2}, \bar{b}_{s}^{\prime}=\bar{b}_{s} / \Gamma_{2}, \bar{b}_{1}^{\prime}=\bar{b}_{1} / \Gamma_{2}, \bar{c}^{\prime}=\bar{c} / \Gamma_{2}, K=K_{3} / \Gamma_{3}$, $d_{1}^{\prime}=2 d_{1} / \Gamma_{3}$, and $d_{2}^{\prime}=d_{2} / \Gamma_{3}$.

We summarize the coefficients in (81)-(83) as follows. $\gamma$ is a damping coefficient in the time evolution equation of $v_{\alpha}$; although the motionless spot is stable for $\gamma<0$, the motionless spot is destabilized for $\gamma>0$ and there appears a moving spot. $\kappa$ and $K$ are damping coefficients in the time evolution equations of $S_{\alpha \beta}$ and $U_{\alpha \beta \gamma}$, respectively. In this study, we fix $K$ as a positive constant and consider $\kappa$ in the range $\kappa>0 . \bar{b}^{\prime}$ and $\bar{b}_{s}^{\prime}$ are coefficients of the quadratic and fourth order of $\mathbf{v}$, respectively, in the time evolution equation of $S_{\alpha \beta}$. The term $\bar{b}_{s}^{\prime}$ can be absorbed into $\bar{b}^{\prime}$ with the replacement $\bar{b}^{\prime} \rightarrow\left(\bar{b}^{\prime}+\bar{b}_{s}^{\prime}|\mathbf{v}|^{2}\right)$. From the results, it can be seen that the term $\bar{b}^{\prime}$ is enhanced (suppressed) when the sign of $\bar{b}_{s}^{\prime}$ is the same as (different from) that of $\bar{b}^{\prime} \cdot d_{1}^{\prime}$ is the coefficient of the cubic term of $\mathbf{v}$ in the time evolution equation of $U_{\alpha \beta \gamma}$. These terms cause deformations with increasing velocity. The other parameters are coupling coefficients of deformation and velocity. $\bar{a}^{\prime}$ and $d_{2}^{\prime}$ are the coupling coefficients of $S_{\alpha \beta} v_{\beta}$ and $S_{\alpha \beta} v_{\gamma}$, respectively, and these terms influence the time evolution of $v_{\alpha}$ and $U_{\alpha \beta \gamma}$, respectively. $\bar{b}_{1}^{\prime}$ and $\bar{c}^{\prime}$ are the coupling coefficients of $U_{\alpha \beta \gamma} v_{\gamma}$ and $|\mathbf{v}|^{2} S_{\alpha \beta}$, respectively. When $\bar{b}_{1}^{\prime}=0, U_{\alpha \beta \gamma}$ is decoupled from $v_{\alpha}$ and $S_{\alpha \beta} \cdot \bar{c}^{\prime}|\mathbf{v}|^{2} S_{\alpha \beta}$ can be absorbed into $-\kappa S_{\alpha \beta}$ with the replacement $\kappa \rightarrow\left(\kappa-\bar{c}^{\prime}|\mathbf{v}|^{2}\right)$. Then, the term $\kappa$ is suppressed (enhanced) when $\bar{c}^{\prime}$ is positive (negative), with $|\mathbf{v}|^{2} \neq 0$.

The dependence of $\bar{a}^{\prime}, \bar{b}^{\prime}, \bar{b}_{s}^{\prime}, \bar{c}^{\prime}, \bar{b}_{1}^{\prime}, d_{1}^{\prime}$, and $d_{2}^{\prime}$ on $k_{c}$ is shown in Figure 4 . In each figure, these parameters (shown on the vertical axis) are scaled by $R_{0}^{2}$. We see that both $\bar{a}^{\prime}$ and $\bar{b}^{\prime}$ are negative, and $\bar{b}_{1}^{\prime}, \bar{c}^{\prime} \neq 0$ even at $k_{c}=0$. Although $\bar{b}_{1}^{\prime}$ is a monotonically increasing function of $k_{c}, \bar{c}^{\prime}$ is a monotonically decreasing function of $k_{c} \cdot d_{1}^{\prime}$ and $d_{2}^{\prime}$ are positive but $d_{1}^{\prime}$ is much smaller than $d_{2}^{\prime}$.

Henceforth, we drop the primes on the parameters in the full tensor model (81)-(83). In the full tensor model, when $K$ is large and $\mathbf{v}$ is small, the effect of $U_{\alpha \beta \gamma}$ on $S_{\alpha \beta}$ is small in (82). In this case, we put $\bar{b}_{1}=0$, and the $v_{\alpha}, S_{\alpha \beta}$, and $U_{\alpha \beta \gamma}$ system described by (81)-(83) is reduced to a $v_{\alpha}$ and $S_{\alpha \beta}$ system. We call this system the reduced tensor model.

\section{Stationary Solution and Phase Diagrams}

In the following subsections, we consider the stationary solution and phase diagrams. For this, we rewrite $v_{\alpha}, S_{\alpha \beta}$, and $U_{\alpha \beta \gamma}$ by introducing the following variables:

$$
\begin{aligned}
v_{1} & =v \cos \phi, \\
v_{2} & =v \sin \phi, \\
S_{11} & =\frac{s}{2} \cos 2 \theta, \\
S_{12} & =\frac{s}{2} \sin 2 \theta, \\
U_{111} & =c_{3}+c_{-3}, \\
U_{222} & =-\mathrm{i}\left(c_{3}-c_{-3}\right), \\
c_{ \pm 3} & =\frac{\delta_{3}}{2} e^{\mp \mathrm{i} 3 \phi_{3}} \equiv \frac{z}{4} e^{\mp \mathrm{i} 3 \varphi},
\end{aligned}
$$

where we choose $v, s, z>0$. From (81), we obtain the time evolution equations of $v$ and $\phi$ as

$$
\begin{aligned}
& \frac{d}{d t} v=v\left(\gamma-v^{2}\right)-\frac{1}{2} \bar{a} s v \cos 2(\theta-\phi), \\
& \frac{d}{d t} \phi=-\frac{1}{2} \bar{a} s \sin 2(\theta-\phi),
\end{aligned}
$$

respectively. From (82), we obtain the time evolution equations of $s$ and $\theta$ as

$$
\begin{aligned}
\frac{d}{d t} s= & -\kappa s+\bar{b} v^{2} \cos 2(\theta-\phi)+\bar{b}_{s} v^{4} \cos 2(\theta-\phi) \\
& +\bar{b}_{1} z v \cos (2 \theta+\phi-3 \varphi)+\bar{c} v^{2} s \\
\frac{d}{d t} \theta= & -\frac{\bar{b} v^{2}}{2 s} \sin 2(\theta-\phi)-\frac{\bar{b}_{s} v^{4}}{2 s} \sin 2(\theta-\phi) \\
& +\frac{\bar{b}_{1} z v}{2 s} \sin (3 \varphi-\phi-2 \theta)
\end{aligned}
$$




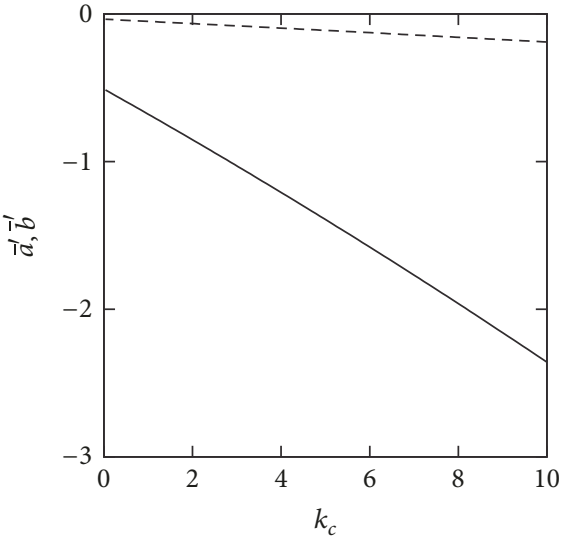

(a)

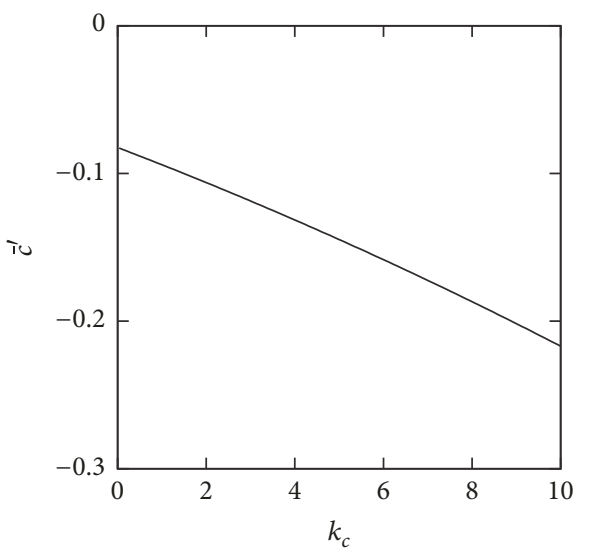

(c)

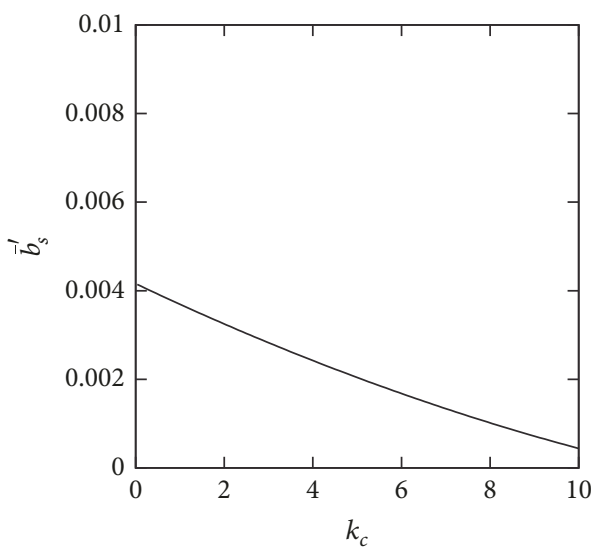

(b)

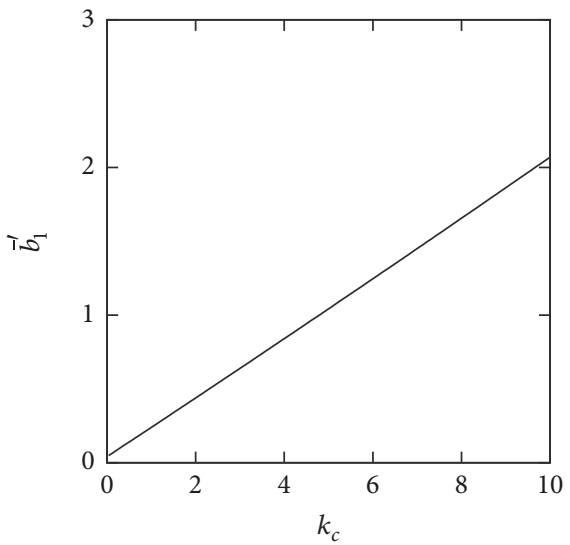

(d)

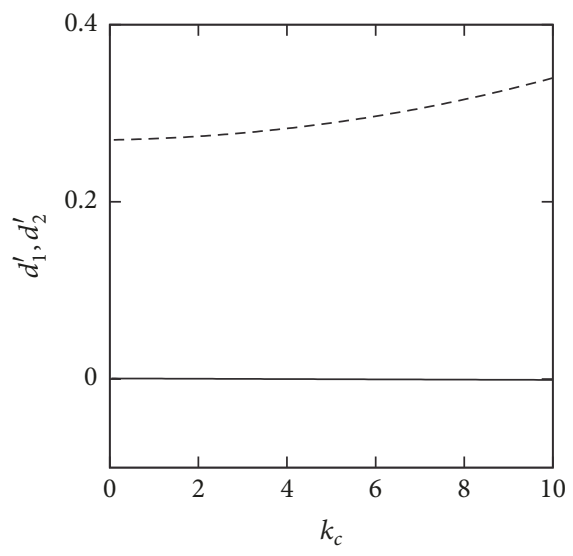

(e)

Figure 4: Dependence of $\bar{a}^{\prime}, \bar{b}^{\prime}, \bar{b}_{s}^{\prime}, \bar{c}^{\prime}, \bar{b}_{1}^{\prime}, d_{1}^{\prime}$, and $d_{2}^{\prime}$ on $k_{c} . \alpha=5.0$ and $W=1.0$. All the parameters in the vertical axis are scaled by $R_{0}^{2}$. (a) $\bar{a}^{\prime}$ and $\bar{b}^{\prime}$. The solid and dashed curves represent $\bar{a}^{\prime}$ and $\bar{b}^{\prime}$, respectively. (b) $\bar{b}_{s}^{\prime}$. (c) $\bar{c}^{\prime}$. (d) $\bar{b}_{1}^{\prime}$. (e) $d_{1}^{\prime}$ and $d_{2}^{\prime}$. The solid and dashed curves represent $d_{1}^{\prime}$ and $d_{2}^{\prime}$, respectively.

respectively. From (83), we obtain the time evolution equations of $z$ and $\varphi$ as

$$
\begin{aligned}
\frac{d}{d t} z= & -K z+d_{1} v^{3} \cos 3(\varphi-\phi) \\
& +d_{2} s v \cos (3 \varphi-2 \theta-\phi),
\end{aligned}
$$

$$
\begin{aligned}
\frac{d}{d t} \varphi= & -\frac{d_{1}}{3 z} v^{3} \sin 3(\varphi-\phi) \\
& -\frac{d_{2}}{3 z} s v \sin (3 \varphi-2 \theta-\phi) .
\end{aligned}
$$

Using (85)-(90), we theoretically derive the stationary solution and phase diagrams. In order to verify the theoretical 
result, we perform simulations of the reduced tensor model. For the numerical calculations, we employ the fourth-order Runge-Kutta algorithm with the time increment $\Delta t=1.0 \times$ $10^{-4}$ and fix the parameters as $\bar{a}=\bar{b}=-1, K=0.3, d_{1}=0.1$, and $d_{2}=0.5$.

6.1. Stationary Solution. For the reduced and full tensor models, we consider the stationary solution of the moving spot, which moves straight in the $x$ direction. This situation corresponds to $\phi=0$. In the case of $\bar{b}_{1}=\bar{b}_{s}=\bar{c}=0$, the stationary solution of (85) and (86) becomes $v_{0}^{2}=\gamma /(1+B)$ with $B=\bar{a} \bar{b} / 2 \kappa$. In order to avoid the divergence of $v_{0}^{2}$, we assume that $\bar{a} \bar{b}>0$. For $\bar{c} \neq 0$, we consider the dependence of the stationary solution of $s, z, \theta$, and $\varphi$ on the signs of $\bar{a}$ and $\bar{b}$. In the stationary state of (86) with $\phi=0$, there are two stationary solutions of $\theta$ depending on the sign of $\bar{a}$ and $\bar{b}$ : (I) $\bar{a}$ and $\bar{b}$ are positive with $\theta_{0}=l \pi$ and (II) $\bar{a}$ and $\bar{b}$ are negative with $\theta_{0}=(l+1 / 2) \pi$, where $l$ is an integer. Using (87)-(90), the stationary solution of case (I) is

$$
\begin{aligned}
s_{0} & =\left[\frac{\left(\bar{b}+\bar{b}_{s} v_{0}^{2}\right) K+\bar{b}_{1} d_{1} v_{0}^{2}}{K\left(\kappa-\bar{c} v_{0}^{2}\right)-d_{2} \bar{b}_{1} v_{0}^{2}}\right] v_{0}^{2}, \\
z_{0} & =\cos 3 \varphi_{0}\left[\frac{d_{1}\left(\kappa-\bar{c} v_{0}^{2}\right)+d_{2}\left(\bar{b}+\bar{b}_{s} v_{0}^{2}\right)}{K\left(\kappa-\bar{c} v_{0}^{2}\right)-d_{2} \bar{b}_{1} v_{0}^{2}}\right] v_{0}^{3},
\end{aligned}
$$

where $\varphi_{0}$ satisfies $\sin 3 \varphi_{0}=0$ and $\nu_{0}$ is a stationary solution of $v$, which is determined later. On the other hand, for case (II), the stationary solution is

$$
\begin{aligned}
& s_{0}=-\left[\frac{\left(\bar{b}+\bar{b}_{s} v_{0}^{2}\right) K+\bar{b}_{1} d_{1} v_{0}^{2}}{K\left(\kappa-\bar{c} v_{0}^{2}\right)-d_{2} \bar{b}_{1} v_{0}^{2}}\right] v_{0}^{2}, \\
& z_{0}=\cos 3 \varphi_{0}\left[\frac{d_{1}\left(\kappa-\bar{c} v_{0}^{2}\right)+d_{2}\left(\bar{b}+\bar{b}_{s} v_{0}^{2}\right)}{K\left(\kappa-\bar{c} v_{0}^{2}\right)-d_{2} \bar{b}_{1} v_{0}^{2}}\right] v_{0}^{3} .
\end{aligned}
$$

From these results, it is seen that $z_{0}$ has the same expression irrespective of the signs of $\bar{a}$ and $\bar{b}$. As $z_{0}$ is positive, $\varphi_{0}$ satisfying $\sin 3 \varphi_{0}=0$ is chosen as

(i) $\varphi_{0}=\frac{2 m}{3} \pi$,

$$
\text { if }\left[\frac{d_{1}\left(\kappa-\bar{c} v_{0}^{2}\right)+d_{2}\left(\bar{b}+\bar{b}_{s} v_{0}^{2}\right)}{K\left(\kappa-\bar{c} v_{0}^{2}\right)-v_{0}^{2} d_{2} \bar{b}_{1}}\right]>0,
$$

(ii) $\varphi_{0}=\frac{(2 m+1)}{3} \pi$,

$$
\text { if }\left[\frac{d_{1}\left(\kappa-\bar{c} v_{0}^{2}\right)+d_{2}\left(\bar{b}+\bar{b}_{s} v_{0}^{2}\right)}{K\left(\kappa-\bar{c} v_{0}^{2}\right)-v_{0}^{2} d_{2} \bar{b}_{1}}\right]<0 .
$$

For both cases ((I) and (II)), the stationary solution of $z_{0}$ is given by

$$
z_{0}=\left|\frac{d_{1}\left(\kappa-\bar{c} v_{0}^{2}\right)+d_{2}\left(\bar{b}+\bar{b}_{s} v_{0}^{2}\right)}{K\left(\kappa-\bar{c} v_{0}^{2}\right)-d_{2} \bar{b}_{1} v_{0}^{2}}\right| v_{0}^{3} .
$$

In both cases ((I) and (II)), by substituting $s_{0}$ into (85) in the stationary state, we obtain two stationary solutions $v_{0, \pm}$ as

$$
\begin{aligned}
& v_{0, \pm}^{2}=\frac{1}{f_{1}}\left[f_{2} \pm \sqrt{f_{3}}\right] \\
& \text { with } f_{1}=2\left[K \bar{c}+d_{2} \bar{b}_{1}-\frac{1}{2} \bar{a}\left(\bar{b}_{1} d_{1}+K \bar{b}_{s}\right)\right], \\
& f_{2}=\left(\frac{1}{2} \bar{a} \bar{b}+\kappa+\gamma \bar{c}\right) K+\gamma d_{2} \bar{b}_{1}, \\
& f_{3}=\left[\left(\frac{1}{2} \bar{a} \bar{b}-\kappa+\gamma \bar{c}\right) K+\gamma d_{2} \bar{b}_{1}\right]^{2}+2 \bar{a} \kappa\left[\left(\bar{b}+\bar{b}_{s} \gamma\right) K^{2}+\gamma \bar{b}_{1} d_{1} K\right] .
\end{aligned}
$$

In order to satisfy the condition that $v_{0, \pm}^{2} \rightarrow \gamma /(1+B)$ in the limits $\bar{b}_{1}, \bar{b}_{s}$, and $\bar{c} \rightarrow 0$, only $v_{0,-}^{2}$ exists. In the physical images of the moving spot in cases (I) and (II) with $\varphi_{0}=0$, the long axes of the elliptical domain are parallel and perpendicular to the moving direction, respectively [16].

6.2. Stability of Straight Motion in the Reduced Tensor Model. When $K$ is large in (89), $z$ is rapidly damped to zero. In addition, when $v$ is small, the influences of $z$ on $s$ and $\theta$ in (87) and (88) are small. For this case, we discuss the stability of the straight motion of the spot by setting $\bar{b}_{1}=0$ in (87) and
(88). Using (86) and (88), we can derive the time evolution equation of $\psi=\theta-\phi$ as

$$
\frac{d \psi}{d t}=-\frac{1}{2}\left(-\bar{a} s+\frac{\bar{b} v^{2}}{s}+\frac{\bar{b}_{s} v^{4}}{s}\right) \sin 2 \psi .
$$

For the stationary states $v=v_{0,-}, s=s_{0}$, we discuss the stability condition of the straight motion. From (96), the stationary solution $\psi_{0}$ satisfies $2 \psi_{0}=2 l \pi$ or $(2 l+1) \pi$, and their corresponding stability conditions are $\left(-\bar{a} s_{0}+\bar{b} v_{0,-}^{2} / s_{0}+\right.$ $\left.\bar{b}_{s} v_{0,-}^{4} / s_{0}\right)>0$ or $\left(-\bar{a} s_{0}+\bar{b} v_{0,-}^{2} / s_{0}+\bar{b}_{s} v_{0,-}^{4} / s_{0}\right)<0$, respectively. Since $\bar{a}$ and $\bar{b}$ are negative in our RD system, as shown in Figure 4(a), we consider the case of $\psi_{0}=\pi / 2$ as follows. 


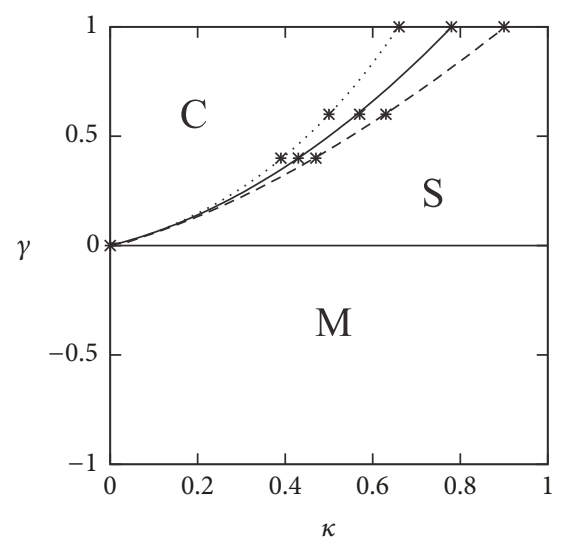

(a)

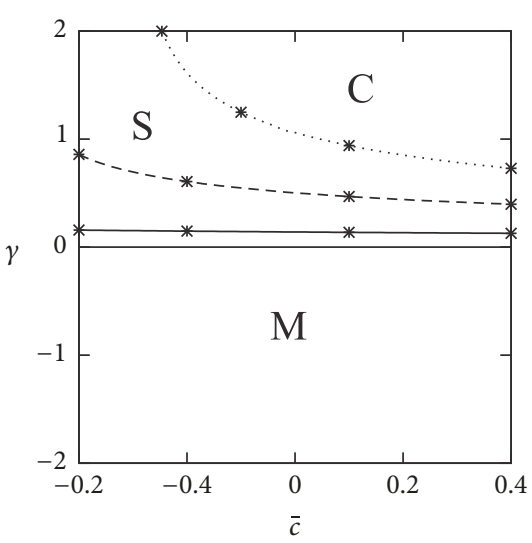

(b)

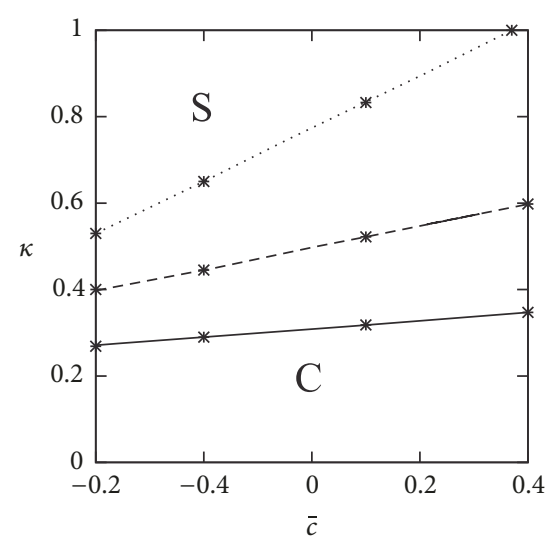

(c)

Figure 5: Phase diagram of spot motion. $\bar{a}=\bar{b}=-1$ and $\bar{b}_{s}=0.05$. Data are obtained by the analysis of (96). M, S, and C in the figure represent regions of no motion, straight motion, and circular motion, respectively. The mark $*$ represents the data obtained by the simulation of the reduced tensor model. (a) Phase diagram in the $\kappa-\gamma$ plane. The solid, dashed, and dotted curves correspond to the cases of $\bar{c}=0,0.2$, and -0.2 , respectively. The upper (lower) region of each curve corresponds to the region of circular (straight) motion, and the region $\gamma \leq 0$ corresponds to the region of no motion. (b) Phase diagram in the $\bar{c}-\gamma$ plane. The solid, dashed, and dotted curves correspond to cases of $\kappa=0.2,0.5$, and 0.8 , respectively. The region across each curve is the same as in (a). (c) Phase diagram in the $\bar{c}-\kappa$ plane. The solid, dashed, and dotted curves correspond to the cases of $\gamma=0.25,0.5$, and 1.0, respectively. The upper (lower) region of each curve corresponds to the region of straight (circular) motion.

In this case, the stability condition is calculated using the $s_{0}$ given by (92). The bifurcation of straight-circular motion, when $\bar{b}_{s}=\bar{c}=0$, was already studied in [16], and the critical value for the straight motion is expressed as

$$
\gamma_{c}=\frac{\kappa^{2}}{\bar{a} \bar{b}}+\frac{\kappa}{2} .
$$

The phase diagram in the $\kappa-\gamma$ plane is shown by the solid curve in Figure 5(a). When $\gamma \leq 0$, the motionless spot is stable. When $\gamma$ is in the range $0<\gamma \leq \gamma_{c}$, the spot moves straight. However, when $\gamma>\gamma_{c}$, the straight motion become unstable and changes into circular motion. On the other hand, when $\bar{b}_{s} \neq 0$ and $\bar{c} \neq 0$, the stability condition for straight motion is

$$
\bar{a}\left[\frac{\bar{b} v_{0,-}^{2}+\bar{b}_{s} v_{0,-}^{4}}{\kappa-\bar{c} v_{0,-}^{2}}\right]<\kappa-\bar{c} v_{0,-}^{2}
$$

The phase diagram is a curved surface in $\kappa-\gamma-\bar{c}$ space; the boundary of different motions of spot is obtained by using (98). The phase diagram in the $\kappa-\gamma$ plane is shown in Figure 5(a) with dashed and dotted curves. Comparing the curves with that for the case when $\bar{b}_{s}=\bar{c}=0$ (the solid curve), it can be seen that when $\bar{c}>0$, the parameter region for the circular motion is larger. However, when $\bar{c}<0$, the parameter region for the circular motion is smaller. We can explain this result using (87); the term $\bar{c} v^{2} s$ essentially reduces the parameter $\kappa$ of the damping term $-\kappa s$ such that the damping term becomes $-\left(\kappa-\bar{c} v^{2}\right) s$. When $\bar{c}$ is positive (negative), the term $\bar{c} v^{2} s$ effectively reduces (increases) $\kappa$ so that the deformation $s$ becomes large (small). This leads to positive (negative) $\bar{c}$ results in the larger parameter region of the circular motion. In our RD system, $\bar{c}$ is negative and $|\bar{c}|$ monotonically increases with $k_{c}$ (see Figure $4(\mathrm{c})$ ), and the parameter region for circular motion becomes smaller as the intensity of chemotaxis increases. The phase diagrams in the $\bar{c}-\gamma$ plane and $\bar{c}-\kappa$ plane are shown in Figures 5(b) and 5(c), respectively. Figure 5(b) suggests that the parameter region for the circular motion is smaller for a larger value of $\kappa$. Equation (87) suggests that a larger value of $\kappa$ damps $s$ strongly such that the deformation is small, resulting in a smaller region of the circular motion. On the other hand, Figure 5(c) suggests that the parameter region for the circular motion is larger for a larger value of $\gamma$. Equation (85) suggests that a large positive value of $\gamma$ enhances $v$ such that the deformation becomes large, resulting in a larger region of circular motion. In each figure, the simulation results for the reduced tensor model are shown; they agree well with the theoretical results.

6.3. Dependence of Critical Velocity on Parameters. In the previous subsection, we showed that a spot appears in the circular motion when $\gamma>\gamma_{c}$. In this parameter region, we examine the stationary circular motion of the spot with a constant angular frequency $\omega$ and velocity $v_{r}$. For this, we set $\bar{b}_{1}=0$ and substitute $v=v_{r}, s=s_{r}, \theta=\omega t+\zeta / 2$, and $\phi=\omega t$ into (85)-(88), and obtain the relations among $\zeta, s_{r}, v_{r}$, and $\omega$. The calculation is straightforward; the final expressions are shown.

We first consider the case when $\bar{b}_{s}=\bar{c}=0$, where up to $O\left(\mathbf{v}^{3}\right)$ terms are considered, and obtain the relations $\cos \zeta=$ $\kappa / \bar{a} s_{r}, s_{r}^{2}=\bar{b} v_{r}^{2} / \bar{a}, v_{r}^{2}=(\gamma-\kappa / 2), \omega^{2}=(\bar{a} \bar{b} / 4)\left(v_{r}^{2}-v_{c}^{2}\right)$, and $v_{c}^{2}=$ $\kappa^{2} / \bar{a} \bar{b}$. When the spot rotates with an angular frequency $\omega, \omega$ satisfies $\omega^{2} \geq 0$, and this condition leads to $v_{r} \geq v_{c}$ [16]. The dependence of the critical velocity $v_{c}$ for the stable angular 


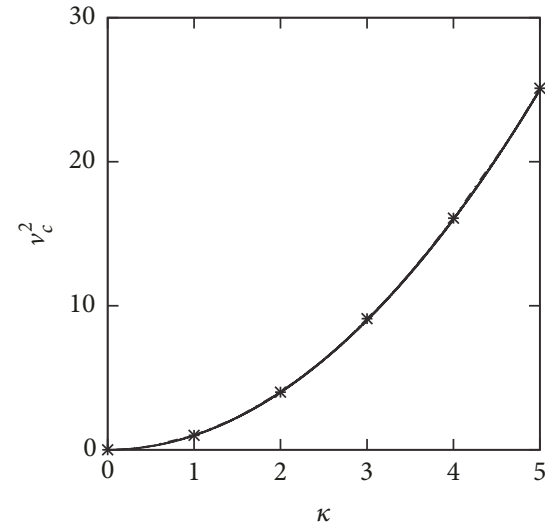

(a)

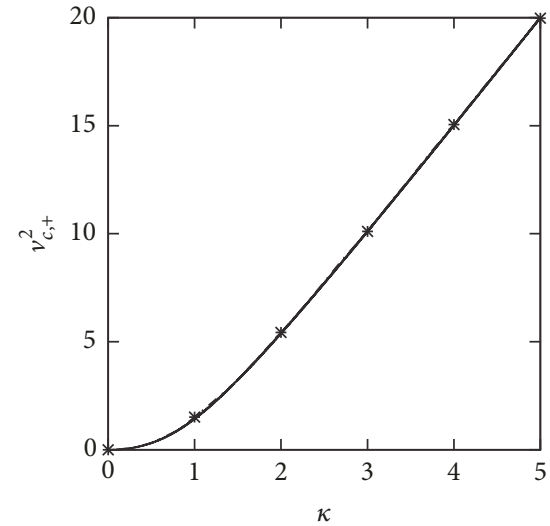

(b)

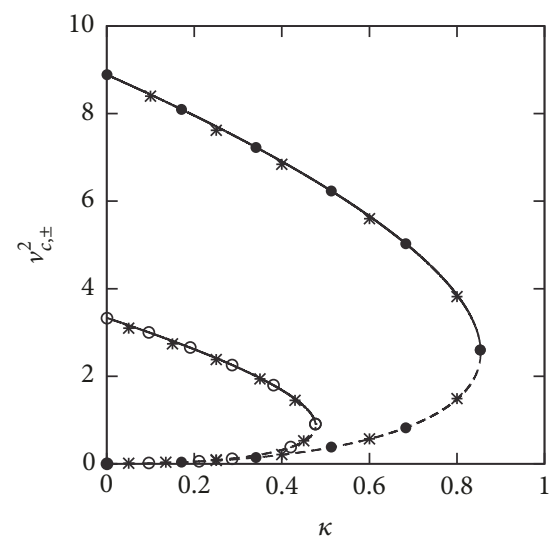

(c)

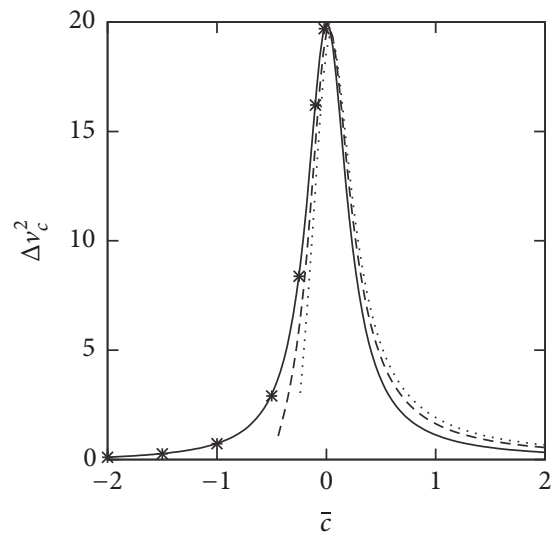

(d)

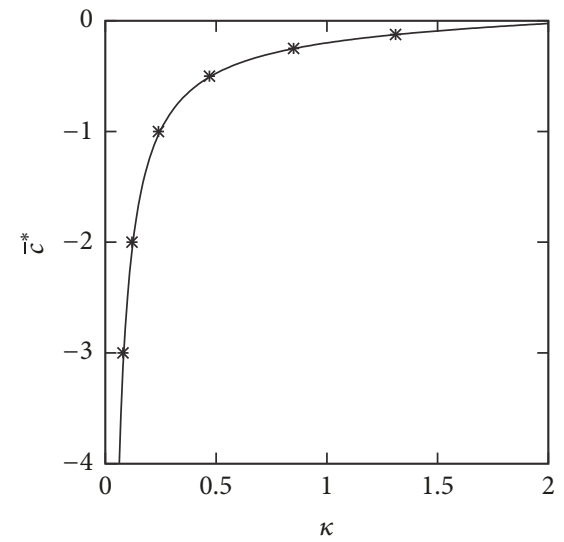

(e)

FIGURE 6: Dependence of the critical velocity $v_{c}, v_{c, \pm}, \Delta v_{c}^{2}$, and $\bar{c}^{*}$ on $\kappa$ and $\bar{c} . \bar{a}=\bar{b}=-1$. The mark $*$ represents data obtained by the simulation of the reduced tensor model. (a) Dependence of $v_{c}^{2}$ on $\kappa \cdot \bar{b}_{s}=\bar{c}=0$. (b) Dependence of $v_{c,+}^{2}$ on $\kappa \cdot \bar{b}_{s}=-0.2$ and $\bar{c}=-0.25$. (c) Dependence of $v_{c, \pm}^{2}$ on $\kappa \cdot \bar{b}_{s}=0.05$. The curves with filled $(\bullet)$ and open ( $\circ$ ) circles correspond to the cases of $\bar{c}=-0.25$ and $\bar{c}=-0.5$, respectively. The solid and dashed curves represent $v_{c,-}^{2}$ and $v_{c,+}^{2}$, respectively. (d) Dependence of $\Delta v_{c}^{2}$ on $\bar{c}$. $\bar{b}_{s}=0.05$. The solid, dashed, and dotted curves correspond to $\kappa=0.1,0.5$, and 0.8 , respectively. (e) Dependence of $\bar{c}^{*}$ on $\kappa . \bar{b}_{s}=0.05$.

frequency on $\kappa(\geq 0)$ is shown in Figure 6(a). In this case, there is a lower limit of velocity $v_{c}$ for the circular motion but no upper limit of velocity for the same.

Next, we consider the case when $\bar{b}_{s} \neq 0$ and $\bar{c} \neq 0$, where up to $O\left(\mathbf{v}^{4}\right)$ terms are considered. After similar calculations to the case of $\bar{b}_{s}=\bar{c}=0$, we obtain the relations $\cos \zeta=$ $\left(\kappa-\bar{c} v_{r}^{2}\right) / \bar{a} s_{r}, s_{r}^{2}=\left(\bar{b} v_{r}^{2}+\bar{b} v_{s}^{4}\right) / \bar{a}, v_{r}^{2}=(\gamma-\kappa / 2) /(1-\bar{c} / 2)$, and $\omega^{2}=\left(\bar{a} \bar{b}_{s}-\bar{c}^{2}\right) v_{r}^{4} / 4+(\bar{a} \bar{b}+2 \kappa \bar{c}) v_{r}^{2} / 4-\kappa^{2} / 4$. In this case, $\omega^{2}$ is a quartic function of $v_{r}$. For the condition $\omega^{2} \geq 0$, there are two cases depending on the sign of $\left(\bar{a}_{s}-\bar{c}^{2}\right)$. When $\left(\bar{a} \bar{b}_{s}-\bar{c}^{2}\right)>0$, $\omega^{2}$ exists for $v_{r}^{2} \geq v_{c,+}^{2}$, where $v_{c, \pm}^{2}$ are the solutions of $\omega^{2}=0$, which are given by $v_{c, \pm}^{2}=\left[-(\bar{a} \bar{b}+2 \kappa \bar{c}) \pm \sqrt{D_{r}}\right] /\left[2\left(\bar{a} \bar{b}_{s}-\bar{c}^{2}\right)\right]$ with $D_{r}=(\bar{a} \bar{b}+2 \kappa \bar{c})^{2}+4 \kappa^{2}\left(\bar{a} \bar{b}_{s}-\bar{c}^{2}\right)$. The dependence of $v_{c,+}^{2}$ on $\kappa$ is shown in Figure 6(b); there is only a lower limit of velocity $v_{c,+}$. This property is the same with the case when $\bar{b}_{c}=\bar{c}=0$. On the other hand, when $\left(\bar{a}_{\bar{s}}-\bar{c}^{2}\right)<0, \omega^{2}$ exists in a certain range of $v_{r}^{2} ; v_{c,+}^{2} \leq v_{r}^{2} \leq v_{c,-}^{2}$ under the condition $D_{r} \geq 0$. In this case, there is a lower limit and an upper limit of velocity, $v_{c,+}$ and $v_{c,-}$, respectively. The gap between $v_{c,-}^{2}$ and $v_{c,+}^{2}$ is defined by $\Delta v_{c}^{2}=v_{c,-}^{2}-v_{c,+}^{2}=\sqrt{D_{r}} /\left(\bar{c}^{2}-\bar{a} \bar{b}_{s}\right)$. Let us consider two limiting cases. In the limit $|\bar{c}| \rightarrow \infty, v_{c, \pm}^{2}$ and $\Delta v_{c}^{2} \rightarrow 0$. That is, the circular motion cannot exist. On the other hand, for small $|\bar{c}|$ and $\left|\bar{b}_{s}\right| \ll 1$, we expand $v_{c, \pm}^{2}$ in terms of $\bar{c}$ and $\bar{b}_{s}$ and obtain $v_{c,+}^{2} \sim \kappa^{2} /(\bar{a} \bar{b}+2 \kappa \bar{c})$ and $v_{c,-}^{2} \sim(\bar{a} \bar{b}+2 \kappa \bar{c}) /\left(\bar{c}^{2}-\bar{a}_{s}\right)$. Thus, in the limits $\bar{b}_{s}$ and $\bar{c} \rightarrow 0$, $v_{c,+}^{2} \rightarrow \kappa^{2} / \bar{a} \bar{b}, v_{c,-}^{2} \rightarrow \infty$, and $\Delta v_{c}^{2} \rightarrow \infty$. This suggests that $v_{c,+} \rightarrow v_{c}$ and there is no upper limit of velocity, which are the same with the case when $\bar{b}_{s}=\bar{c}=0$. From the numerical results shown in Figures $4(\mathrm{a}), 4(\mathrm{~b})$, and $4(\mathrm{c}),\left(\bar{a}_{\bar{s}}-\bar{c}^{2}\right)<0$ in our $\mathrm{RD}$ system; therefore, it turns out that $v_{r}^{2}$ exists only in a finite range, $v_{c,+}^{2} \leq v_{r}^{2} \leq v_{c,-}^{2}$. For larger $|\bar{c}|$, the gap $\Delta v_{c, \pm}^{2}$ is smaller, as shown in Figure 6(c). The dependence of $\Delta v_{c}^{2}$ on $\bar{c}$ is shown in Figure 6(d), which suggests that $\Delta v_{c}^{2}$ monotonically decreases with increasing $|\bar{c}|$. Since $|\bar{c}|$ increases monotonically as $k_{c}$ increases (Figure $4(\mathrm{c})$ ), $\Delta v_{c}^{2}$ 


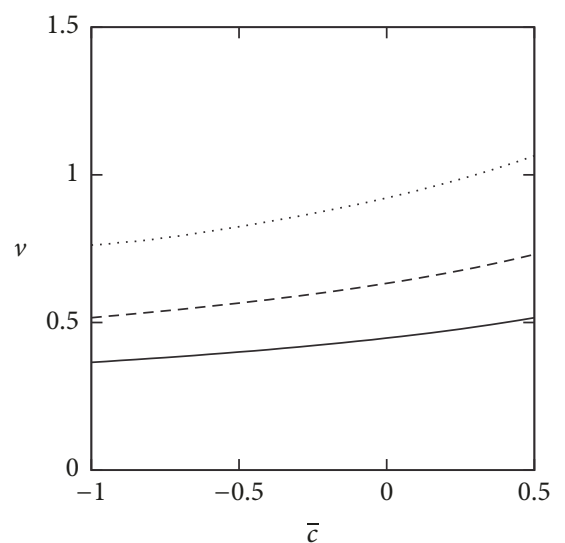

(a)

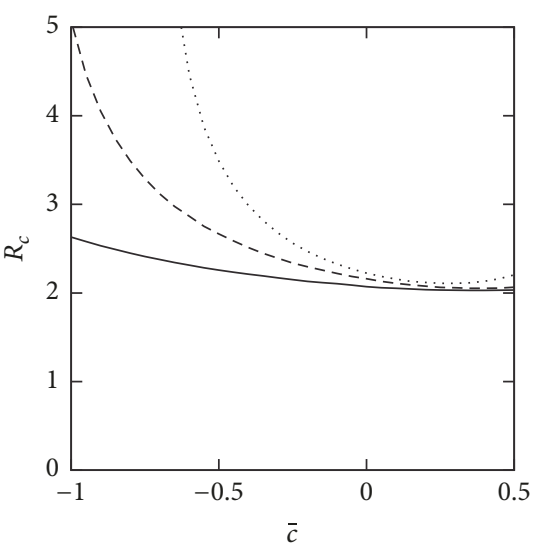

(b)

Figure 7: Dependence of velocity and radius on $\bar{c}$ in the stationary circular motion. Data are obtained by the simulation of the reduced tensor model. $\bar{b}_{s}=0.1$. The solid, dashed, and dotted curves correspond to $(\gamma, \kappa)=(0.25,0.1),(0.5,0.2)$, and $(1.0,0.3)$, respectively. (a) Dependence of $v$ on $\bar{c}$. (b) Dependence of $R_{c}$ on $\bar{c}$.

decreases as the chemotaxis increases. When the condition $D_{r}=0$ is satisfied, $v_{c,-}=v_{c,+}$ and $\Delta v_{c}^{2}=0$, which yields the critical value $\bar{c}^{*}$ as

$$
\bar{c}^{*}=-\frac{(\bar{a} \bar{b})^{2}+4 \kappa^{2}\left(\bar{a}_{s}\right)}{4 \kappa \bar{a} \bar{b}} .
$$

When $|\bar{c}|>\left|\bar{c}^{*}\right|, D_{r}<0$, and, therefore, $v_{c, \pm}^{2}$ does not exist. There is no parameter region of $\gamma$ for a positive value of $\kappa$; the circular motion does not occur for any values of $\gamma$. The dependence of $\bar{c}^{*}$ on $\kappa$ is shown in Figure 6(e). Equation (99) can be positive for large $\kappa$. However, when $\bar{c}$ is chosen as a positive value, the velocities $v$ and $s$ increase monotonically with increasing $\gamma$, and, therefore, the system diverges in the simulation. For this reason, $\bar{c}^{*}$ is shown in the range $\bar{c}^{*} \leq 0$.

In order to verify the above theoretical results, the simulation results for the reduced tensor model are shown in each figure. The results obtained via simulation agree well with the theoretical results. In Figures 6(a) and 6(b), for given $\kappa$ and $\bar{c}$, there is only a lower limit of $\gamma$ for the circular motion of the spot, resulting in $v_{c}$ and $v_{c,+}$, respectively. On the other hand, in Figure 6(c), for given $\kappa$ and $\bar{c}$, there is not only a lower limit of $\gamma$ but also an upper limit of $\gamma$ for the circular motion of the spot, resulting in $v_{c,+}$ and $v_{c,-}$, respectively. However, in Figure 6(e), for given $\kappa$ and $\bar{c}^{*}$, there is no parameter region of $\gamma$ for the circular motion of the spot.

The dependence of the stationary velocity and radius of the circular motion on $\bar{c}$ are shown in Figure 7 . The velocity $v$ increases monotonically with increasing $\bar{c}$, as shown in Figure 7(a). The corresponding radius of the spot motion $R_{c}$ is shown in Figure 7(b). For fixed values of $\gamma$ and $\kappa, v$ decreases and $R_{c}$ increases as $|\bar{c}|$ increases in the range $\bar{c} \leq 0$. The effect of chemotaxis is incorporated in $\bar{c}$, and $\bar{c}$ is negative; $|\bar{c}|$ increases as the intensity of chemotaxis $k_{c}$ increases in our RD system (Figure 4(c)). Thus, it is seen that the chemotaxis reduces $v$ and increases $R_{c}$.
From the above analyses on (85)-(88) with $\bar{b}_{1}=0$, we conclude four points on the properties of stationary circular motion. (i) There is a lower limit of velocity. (ii) Although there is no upper limit for the velocity when up to $O\left(v^{3}\right)$ terms are considered, there will be an upper limit for the velocity if up to $O\left(\mathbf{v}^{4}\right)$ terms are considered. (iii) The range of velocity decreases as the chemotaxis increases. (iv) There is a critical value $\bar{c}^{*}$ (corresponding critical intensity of the chemotaxis is denoted by $\left.k_{c}^{*}\right)$ such that when $|\bar{c}|>\left|\bar{c}^{*}\right|\left(k_{c}>k_{c}^{*}\right)$, the circular motion does not occur for any values of $\gamma$.

6.4. Simulation of the Full Tensor Model. In this subsection, we examine the effect of $U_{\alpha \beta \gamma}$ on $v_{\alpha}$ and $S_{\alpha \beta}$. For this, we consider the case of $\bar{b}_{1} \neq 0$. The data are obtained by the simulation of the full tensor model.

The phase diagrams of the spot motion are shown in Figure 8 . The phase diagrams in the $\bar{c}-\gamma$ plane and $\bar{c}-\kappa$ plane are shown in Figures $8(\mathrm{a})$ and $8(\mathrm{~b})$, respectively. We note that the behaviors in the full tensor model are similar to that in the reduced model (Figures 5(b) and 5(c)). For positive (negative) $\bar{b}_{1}$, the region of the circular motion is larger (smaller). The phase diagrams in the $\bar{b}_{1}-\gamma$ plane and $\bar{b}_{1}-\kappa$ plane are shown in Figures $8(\mathrm{c})$ and $8(\mathrm{~d})$, respectively. The behaviors are similar to the phase diagrams in the $\bar{c}-\gamma$ plane and $\bar{c}-\kappa$ plane. This result suggests that the effect of $\sum_{\gamma} U_{\alpha \beta \gamma} v_{\gamma}$ on the time evolution equation of $S_{\alpha \beta}$ is similar to that of $|\mathbf{v}|^{2} S_{\alpha \beta}$.

The phase diagram of spot motion in the $\bar{c}-\bar{b}_{1}$ plane is shown in Figure 9. It can be seen that the circular motion occurs in the parameter region of positive and large values of $\bar{c}$ and $\bar{b}_{1} . S_{\alpha \beta}$ and $U_{\alpha \beta \gamma}$ measure the elliptical deformation and head-tail asymmetric deformation of the spot, respectively; these deformations lead to the circular motion.

The effects of $\bar{c}$ and $\bar{b}_{1}$ on the stationary velocity $v$ and the corresponding radius of circular motion $R_{c}$ are shown in Figure 10. On comparing these figures, it can 


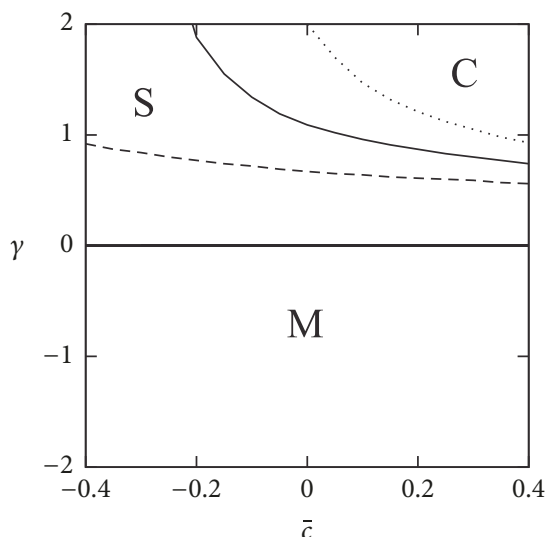

(a)

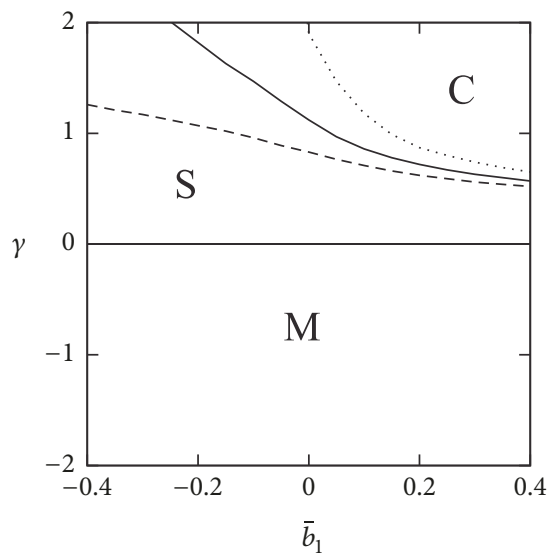

(c)

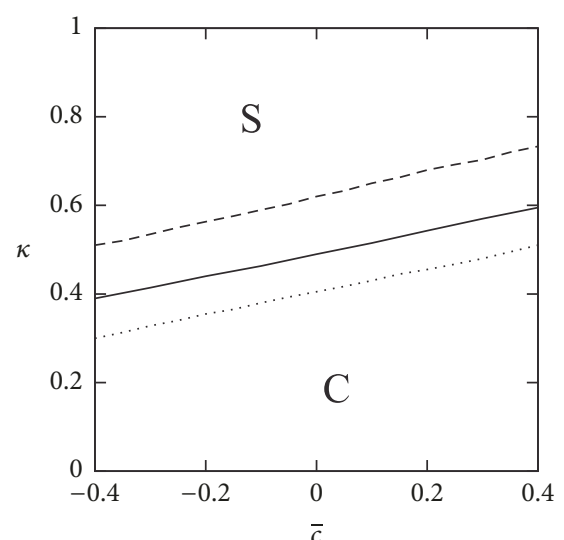

(b)

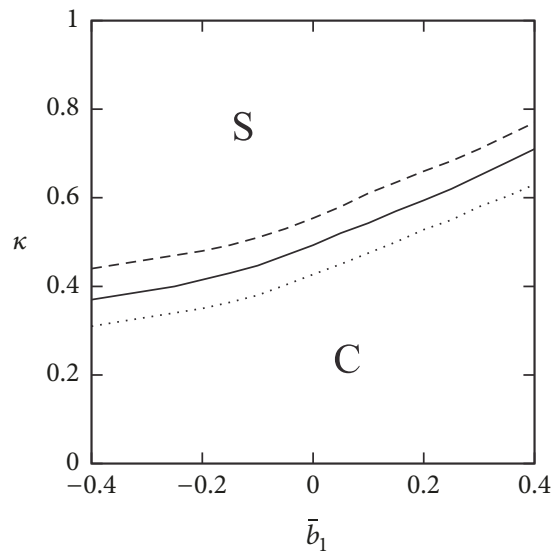

(d)

FIGURE 8: Phase diagram of spot motion. Data are obtained by the simulation of the full tensor model. $\bar{b}_{s}=0.1 . \mathrm{M}, \mathrm{S}$, and C in the figure represent regions of no motion, straight motion, and circular motion, respectively. (a) Phase diagram in the $\bar{c}-\gamma$ plane. $\kappa=0.8$. The solid, dashed, and dotted curves correspond to the cases of $\bar{b}_{1}=0,0.25$, and -0.25 , respectively. The upper (lower) region of each curve corresponds to the region of circular (straight) motion, and the region $\gamma \leq 0$ corresponds to the region of no motion. (b) Phase diagram in the $\bar{c}-\kappa$ plane. $\gamma=0.5$. The solid, dashed, and dotted curves correspond to the cases of $\bar{b}_{1}=0,0.25$, and -0.25 , respectively. The upper (lower) region of each curve corresponds to the region of straight (circular) motion. (c) Phase diagram in the $\bar{b}_{1}-\gamma$ plane. $\kappa=0.8$. The solid, dashed, and dotted curves correspond to the cases of $\bar{c}=0,0.25$, and -0.25 , respectively. The region across each curve is the same as in (a). (d) Phase diagram in the $\bar{b}_{1}-\kappa$ plane. $\gamma=0.5$. The solid, dashed, and dotted curves correspond to the cases of $\bar{c}=0,0.25$, and -0.25 , respectively. The region across each curve is the same as in (b).

be seen that although $v$ and $R_{c}$ depend on $\bar{c}$ and $\bar{b}_{1}$, the influences of $\bar{b}_{1}$ on $v$ and $R_{c}$ are much smaller than those of $\bar{c}$.

The dependence of the critical velocity $v_{c, \pm}$ and $\bar{c}^{*}$ on $\kappa$ is shown in Figure 11. There exists a critical velocity $v_{c \pm}$ for $\bar{b}_{1} \neq$ 0 as shown in Figure 11(a). For larger $\bar{b}_{1}$, the gap $\Delta v_{c}^{2}$ is larger. This suggests that $\bar{b}_{1} \sum_{\gamma} U_{\alpha \beta \gamma} v_{\gamma}$ enhances the circular motion, which is consistent with the results shown in Figure 8. For positive $\bar{b}_{1},\left|\bar{c}^{*}\right|$ becomes larger than that in the reduced tensor model (solid curve) as shown in Figure 11(b). On the other hand, for negative $\bar{b}_{1}$, there is no $\bar{c}^{*}$. In our system, $\bar{b}_{1}$ is positive and increases as the intensity of chemotaxis $k_{c}$ increases as shown in Figure 4(d). Thus, $\bar{c}^{*}$ still exists in the full tensor model, and $\left|\vec{c}^{*}\right|$ is larger than that in the reduced model.

\section{Discussions}

In this section, we discuss the physical origins of the braking effect observed in the previous section. For our RD system, we derived the time evolution equation of $S_{\alpha \beta}$ up to $O\left(\mathbf{v}^{4}\right)$ (see (77)). There were three terms of $O\left(\mathbf{v}^{4}\right): \bar{b}_{s}\left(v_{\alpha} v_{\beta}-\right.$ $\left.\delta_{\alpha \beta}|\mathbf{v}|^{2} / 2\right)|\mathbf{v}|^{2}, \bar{b}_{1} \sum_{\gamma} U_{\alpha \beta \gamma} v_{\gamma}$, and $\bar{c}|\mathbf{v}|^{2} S_{\alpha \beta}$. The $\bar{b}_{s}$ term can be absorbed into the $\bar{b}\left(v_{\alpha} v_{\beta}-\delta_{\alpha \beta}|\mathbf{v}|^{2} / 2\right)$ term by replacing $\bar{b}$ with $\left(\bar{b}+\bar{b}_{s}|\mathbf{v}|^{2}\right)$. Then, with increasing velocity, the $\bar{b}_{s}$ term changes the value of only $\bar{b}$. In our theoretical analysis, considering that the influence of $U_{\alpha \beta \gamma}$ is small, we set $\bar{b}_{1}=$ 0 and examined the effect of $\bar{c}|\mathbf{v}|^{2} S_{\alpha \beta}$ on the stationary solution in Sections 6.2 and 6.3. When up to $O\left(\mathbf{v}^{3}\right)$ terms were considered, there was no upper limit of velocity for 


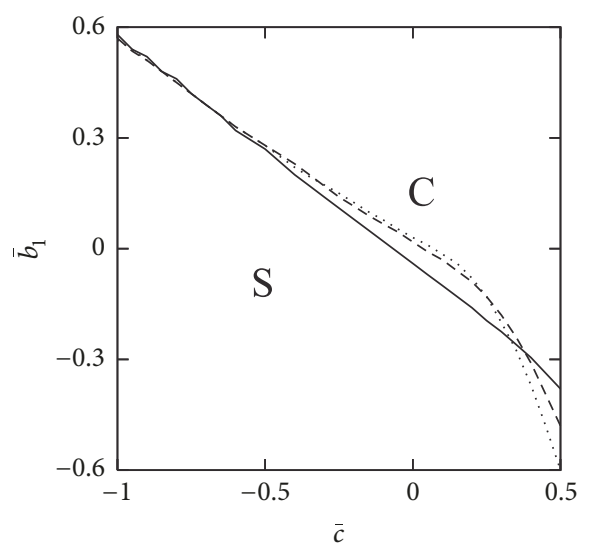

FIGURE 9: Phase diagram of spot motion in the $\bar{c}-\bar{b}_{1}$ plane. Data are obtained by the simulation of the full tensor model. $\bar{b}_{s}=0.1$. The marks $\mathrm{S}$ and $\mathrm{C}$ in the figure represent regions of straight motion and circular motion, respectively. The solid, dashed, and dotted curves correspond to the cases of $(\gamma, \kappa)=(0.25,0.3),(0.5,0.5)$, and $(1.0,0.8)$, respectively.

the circular motion of the spot, as shown in Figure 6(a). However, on including the $O\left(\mathbf{v}^{4}\right)$ terms, even in the absence of chemotaxis $\left(k_{c}=0\right), \bar{c} \neq 0$. The term $\bar{c}|\mathbf{v}|^{2} S_{\alpha \beta}$ influenced the upper limit of velocity of the circular motion, as shown in Figure 6(c). One of the physical origins of the upper limit of velocity is the refractory period behind the rear interface. When $k_{c}=0$, the RD system described by (5) and (6) is considered to have an activator and an inhibitor, as reported by Krischer and Mikhailov [32]. When $\tau$ is large, the motionless pulse (localized domain) in one dimension is stable. With decreasing $\tau$, the motionless pulse is destabilized into a traveling pulse in one dimension. For a traveling pulse in one dimension, there is a refractory period behind the rear interface. A repulsive interaction occurs between pulses in the wave train; the repulsion results from the refractory period imposed on the leading interface of a traveling pulse by the tail of the preceding pulse [38]. In two dimensions, when a spot moves along a circle, there is an upper limit of velocity due to the refractory period behind the rear interface; the refractory period causes a braking effect on the velocity of the spot traveling along a circle.

In the absence of chemotaxis, that is, $k_{c}=0$, we estimate the upper limit of velocity. Let us consider the traveling pulse with velocity $c$ in one dimension of the system described by (5) and (6) in the limit $\epsilon \rightarrow 0$. The spatial dependence of the inhibitor before the leading interface and that behind the rear interface are given by $v_{\text {lead }}(z)=C_{\text {lead }} \exp \left(\lambda_{-} z\right)(z>0)$ and $v_{\text {rear }}(z)=C_{\text {rear }} \exp \left(\lambda_{+} z\right)(z<0)$, respectively, where $z=$ $x-c t, \lambda_{ \pm}=\left[-c \pm \sqrt{c^{2}+4 \beta}\right] / 2$, and $C_{\text {lead(rear) }}$ is a coefficient. Using these expressions, we estimate the relaxation length (refractory period) of the inhibitor behind the rear interface by the linear approximation of $1 / \lambda_{+} \sim c / \beta$ for large $c$. For the spot moving along a circle with a given radius $r_{0}$, the upper limit of velocity $c_{\max }$ will approximately satisfy the relation $2 \pi r_{0} \gg 1 / \lambda_{+} \sim c_{\max } / \beta: c_{\max } \ll 2 \pi r_{0} \beta$. This suggests that, for large $c$, the refractory period is proportional to $c$. As the velocity of the spot increases, the inhibitor at the leading interfaces becomes large due to the overlap, and, therefore, the velocity becomes small (because of the first term in (16)). These effects on velocity are manifested when the spot moves rapidly along a circle with a small radius.

In the presence of chemotaxis, that is, $k_{c}>0$, we discuss another physical origin of the braking effect. The chemotaxis is caused by the gradient of the chemotactic substance at the interface. In our RD system, the chemotactic substance is autosecretion; it is produced inside the spot and diffuses outward. The chemotactic substance is distributed around the center of the spot and monotonically decreases away from the center. For the traveling pulse, the chemotactic substance at the rear interface is larger than that at the leading interface. The gradient of the chemotactic substance at the leading (rear) interface is negative (positive). The absolute value of the gradient of the chemotactic substance at the leading interface is larger than that at the rear interface. In addition, $(d \chi / d v)$ at the leading and rear interfaces are positive. Overall, the chemotactic velocity is negative (because of the second term in (16)). Thus, the chemotaxis in our system essentially reduces velocity.

\section{Conclusions}

In this study, we considered the motion of a spot solution in two dimensions under the influence of chemotaxis. Starting from a three-component RD system, we proposed a twocomponent (an activator and a chemotactic substance) RD system with a global coupling term. We remark that, in our model system, the spot secretes the chemotactic substance from the inside and the motion of the spot is influenced by the chemotaxis. Thus, the model is an autonomous system. The chemotaxis term is of the Keller-Segel type, and the chemotactic velocity is opposite to the traveling direction. The reason for the opposite direction is that the system involves autosecretion, and the gradient of the chemotactic substance at the leading interface is negative. Although there have been several studies on the motion of spots under the influence of chemotaxis, the chemoattractant was supplied from the outside [20-23]. The spot in these models is driven toward the source (higher concentration) point of the chemoattractant. These models are nonautonomous systems, and, therefore, different from our model.

For the RD system, by employing the method proposed in [16], we derived the equation of motion of the spot and the time evolution equations of the tensors. Terms up to the fourth order of $\mathbf{v}$ were considered, and we found that the terms $|\mathbf{v}|^{2} S_{\alpha \beta}$ and $\sum_{\gamma} U_{\alpha \beta \gamma} v_{\gamma}$ played an important role in the motion of the spot. Our numerical results suggested the existence of an upper limit of velocity for the circular motion of the spot due to the braking effect. There are two physical origins for the braking effect: the refractory period behind the rear interface and the chemotactic velocity opposite to the moving direction. The former is unique to the circular motion and is not applicable for a spot in straight motion. On the other hand, the latter is general in our autonomous chemotactic system and is applicable 


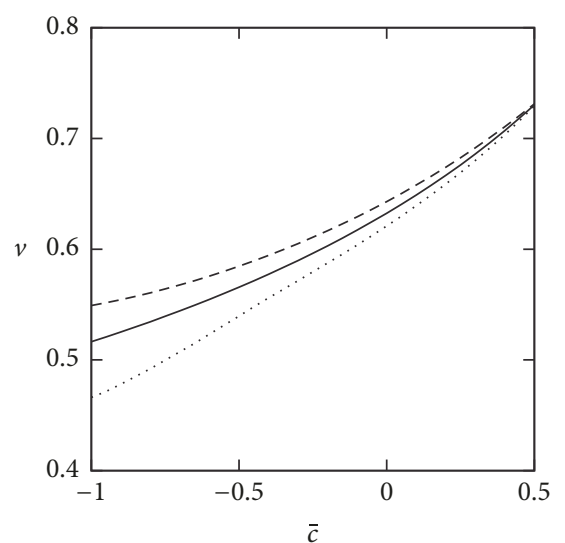

(a)

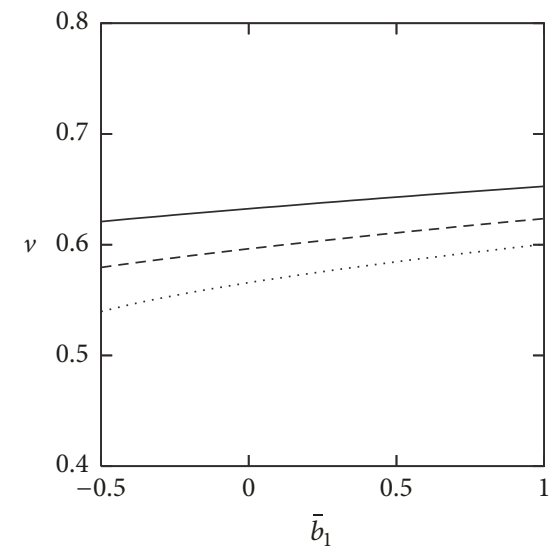

(c)

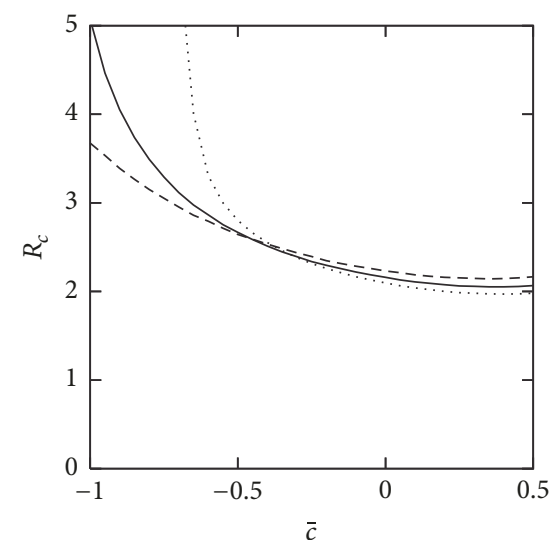

(b)

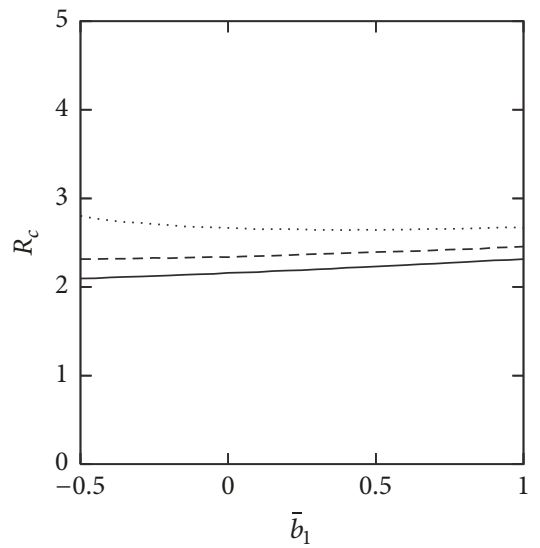

(d)

FIGURE 10: Dependence of velocity and radius on $\bar{c}$ and $\bar{b}_{1}$ in the stationary circular motion. Data are obtained by the simulation of the full tensor model. $\bar{b}_{s}=0.1, \gamma=0.5$, and $\kappa=0.2$. (a) Dependence of $v$ on $\bar{c}$. (b) Dependence of $R_{c}$ on $\bar{c}$, where, in (a) and (b), the solid, dashed, and dotted curves correspond to $\bar{b}_{1}=0,0.5$, and -0.5 , respectively. (c) Dependence of $v$ on $\bar{b}_{1}$. (d) Dependence of $R_{c}$ on $\bar{b}_{1}$, where, in (c) and (d), the solid, dashed, and dotted curves correspond to $\bar{c}=0,-0.25$, and -0.5 , respectively.

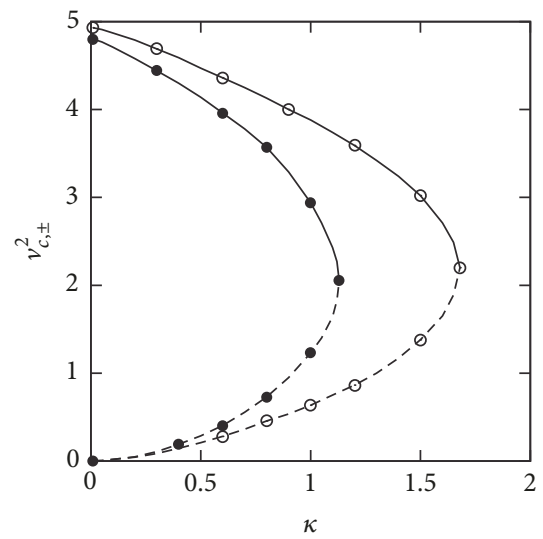

(a)

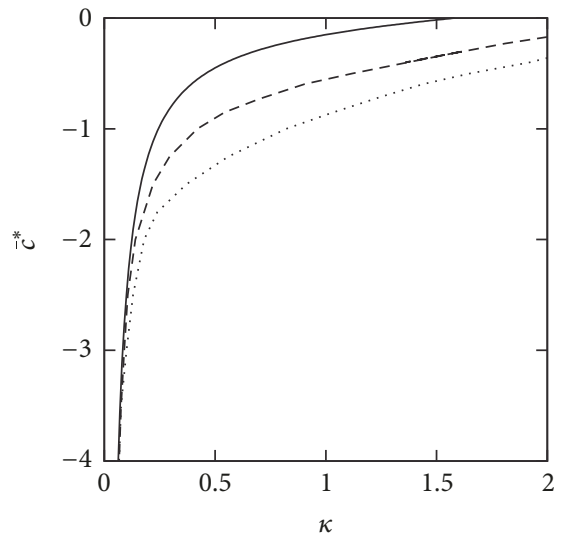

(b)

Figure 11: Dependence of the critical velocity $v_{c, \pm}$ and $\bar{c}^{*}$ on $\kappa$. Data are obtained by the simulation of the full tensor model. $\bar{b}_{s}=0.1$. (a) Dependence of $v_{c, \pm}^{2}$ on $\kappa \cdot \bar{c}=-0.5$. The curves with filled $(\bullet)$ and open $(\circ)$ circles correspond to the cases of $\bar{b}_{1}=0.25$ and $\bar{b}_{1}=0.5$, respectively. The solid and dashed curves represent $v_{c,-}^{2}$ and $v_{c,+}^{2}$, respectively. (b) Dependence of $\bar{c}^{*}$ on $\kappa \cdot \bar{b}_{s}=0.1$. The solid, dashed, and dotted curves correspond to the cases of $\bar{b}_{1}=0,0.25$, and 0.5 , respectively. 
for all moving directions. Owing to the former reason, an upper limit of velocity for the spot moving in a circle exists even in the absence of chemotaxis. Based on the results of our chemotaxis system, we conclude three points. (i) A spot moving on a straight line is destabilized to circular motion with increasing velocity. (ii) The velocity of a spot moving along a circle is restricted to a certain range, which is narrower than that in the absence of the chemotaxis. (iii) There is a critical intensity of chemotaxis $k_{c}^{*}$, and the spot does not undergo circular motion for $k_{c}>k_{c}^{*}$.

In practical experiments, the candidates of autonomous systems including chemotaxis are E. coli and macrophage $[39,40]$. However, there is an essential difference between these biological systems and our model system; E. coli is a rod-shaped cell with a flagellum and macrophage has an amoeba-like shape with pseudopods, and the shape of the motionless state of these cells is not described by a circle. $E$. coli swims by rotating the flagellum, and macrophage crawls by changing its size and shape. In contrast, in our model system, the spot was a solution of an RD system and the shape of the motionless state was assumed to be a circle. The motionless spot was primarily destabilized through drift bifurcation, and it changed into a moving spot with straight motion. The straight moving spot was secondly destabilized through rotation bifurcation, and it changed into a moving spot with circular motion. Due to these differences, the bifurcation points of the straight and circular motions and the critical velocity for the circular motion in these biological systems must be different from those of our model system. However, the collective motions of bacteria and amoebas are modeled by RD systems, and many spatiotemporal patterns are shown [41, 42]: the diffusion-limited aggregation, Edenlike, uniform disk, branched, target, spiral patterns, and so on. This suggests that the behavior of individual cell in these biological systems can be described by $\mathrm{RD}$ systems. From these considerations, the above biological systems are promising candidates of autonomous systems including chemotaxis, and experimental observations of moving cell with circular motion are expected.

\section{Appendix}

\section{A. Velocity of the Interface in One Dimension}

Here, we derive the velocity of the interface in one dimension, given by (16), by following [43]. We denote $h$ as the value of $v$ at the interface $x=x_{0}$ at time $t=t_{0}$. Applying the stretching transformations $\eta=\left(t-t_{0}\right) / \epsilon$ and $\xi=\left(x-x_{0}\right) / \epsilon$ to (5), we obtain

$$
\begin{gathered}
\tau u_{\eta}=u_{\xi \xi}-\left.\left.\left(\frac{d \chi}{d v}\right)\right|_{i}\left(\frac{d v}{d x}\right)\right|_{i} u_{\xi}-u \\
+H(u-p)-h
\end{gathered}
$$

where $\left.(A)\right|_{i}$ denotes the function $A$ evaluated at $x=x_{0}$, and the position of the interface in the stretching coordinate is $\xi=0$. In order to consider the traveling front solution of (A.1), we introduce the moving coordinate $z=\xi-c \eta$. Then, (A.1) becomes

$$
\begin{aligned}
\tau u_{\eta}= & u_{z z}+\left(\tau c-\left.\left.\left(\frac{d \chi}{d v}\right)\right|_{i}\left(\frac{d v}{d x}\right)\right|_{i}\right) u_{z}-u \\
& +H(u-p)-h \\
\equiv & u_{z z}+c^{\prime} u_{z}-u+H(u-p)-h,
\end{aligned}
$$

where $c$ is the velocity of the front and $c^{\prime}=(\tau c-$ $\left.\left.\left.(d \chi / d v)\right|_{i}(d v / d x)\right|_{i}\right)$. The boundary conditions at $z= \pm \infty$ are

$$
\begin{aligned}
& u(-\infty, \eta)=1-h, \\
& u(+\infty, \eta)=-h
\end{aligned}
$$

and the boundary conditions at $z=0$ are

$$
\begin{aligned}
& u(0+, \eta)=u(0-, \eta)=p \\
& u_{z}(0+, \eta)=u_{z}(0-, \eta)
\end{aligned}
$$

The stationary solution of (A.2) is

$$
u(z)= \begin{cases}C_{-} e^{\lambda_{+} z}+(1-h) & (z<0), \\ C_{+} e^{\lambda_{-} z}-h & (z \geq 0),\end{cases}
$$

where $C_{ \pm}$is a coefficient and $\lambda_{ \pm}=(1 / 2)\left[-c^{\prime} \pm \sqrt{c^{\prime 2}+4}\right]$. Under the boundary conditions (A.4), we get $C_{+}=\lambda_{+} /\left(\lambda_{+}-\right.$ $\left.\lambda_{-}\right)=p+h$ and $C_{-}=\lambda_{-} /\left(\lambda_{+}-\lambda_{-}\right)=p+h-1$. Using these relations, we obtain

$$
\begin{aligned}
c^{\prime} & =\tau c-\left.\left.\left(\frac{d \chi}{d v}\right)\right|_{i}\left(\frac{d v}{d x}\right)\right|_{i} \\
& =\frac{2(1 / 2-p-h)}{[(p+h)(1-p-h)]^{1 / 2}} .
\end{aligned}
$$

In the main text, $\tau c$ in (A.6) is denoted as $\tau C(h)$, which is explicitly expressed as (16).

\section{B. Explict Expressions of $h_{0}^{(0)}$ and $\widetilde{h}_{0}^{(0)}$ in the Limit $\epsilon \rightarrow 0$}

We show that in the limit $\epsilon \rightarrow 0, h_{0}^{(0)}$ and $\widetilde{h}_{0}^{(0)}$ calculated using (13) agree with the ones calculated using (34) and (35) with the substitutions $H_{\mathbf{q}}=H_{\mathbf{q}}^{(0)}$ and $\mathbf{R}=\mathbf{R}^{(0)}$, where $\mathbf{R}^{(0)}=R_{0} \mathbf{e}_{r}$, respectively.

$h_{0}^{(0)}$ and $\widetilde{h}_{0}^{(0)}$ obtained directly from (13) are

$$
h_{0}^{(0)}=\bar{v}\left(R_{0}\right)=\frac{1}{\beta}-\frac{R_{0}}{\sqrt{\beta}} K_{1}\left(\sqrt{\beta} R_{0}\right) I_{0}\left(\sqrt{\beta} R_{0}\right)
$$




$$
\widetilde{h}_{0}^{(0)}=\frac{d \bar{v}\left(R_{0}\right)}{d r}=-R_{0} I_{1}\left(\sqrt{\beta} R_{0}\right) K_{1}\left(\sqrt{\beta} R_{0}\right),
$$

respectively.

We first derive $h_{0}^{(0)}$ from the definition (34). Substituting $H_{\mathbf{q}}=H_{\mathbf{q}}^{(0)}$ and $\mathbf{R}=\mathbf{R}^{(0)}$ into (34), we obtain

$$
\begin{aligned}
h_{0}^{(0)} & =\int_{\mathbf{q}} G_{q} H_{\mathbf{q}}^{(0)} e^{\mathrm{iq} \cdot \mathbf{R}^{(0)}} \\
& =R_{0} \int_{0}^{\infty} d q\left(\frac{1}{q^{2}+\beta}\right) J_{0}\left(q R_{0}\right) J_{1}\left(q R_{0}\right) .
\end{aligned}
$$

We apply the formulas of the integrals ([44])

$$
\begin{gathered}
\int_{0}^{\infty} d x \frac{x^{\mu+1}}{\left(x^{2}+t^{2}\right)\left(x^{2}+y^{2}\right)^{v / 2}} J_{\nu}\left(a \sqrt{x^{2}+y^{2}}\right) J_{\mu}(b x) \\
=\frac{t^{\mu} J_{\nu}\left(a \sqrt{y^{2}-t^{2}}\right)}{\left(y^{2}-t^{2}\right)^{v / 2}} K_{\mu}(b t) \\
(b \geq a>0, \quad \operatorname{Re}(\mu) \mid<\operatorname{Re}(v)+2), \\
\int_{0}^{\infty} d x J_{0}(a x) J_{1}(b x)= \begin{cases}\frac{1}{b} & (b>a>0), \\
\frac{1}{2 b} & (b=a>0), \\
0 & (a>b>0)\end{cases}
\end{gathered}
$$

to (B.3); we can confirm that (B.3) agrees with (B.1).

Next, we derive $\widetilde{h}_{0}^{(0)}$ from the definition (35). Substituting $H_{\mathbf{q}}=H_{\mathbf{q}}^{(0)}$ and $\mathbf{R}=\mathbf{R}^{(0)}$ into (35), we obtain

$$
\begin{aligned}
\widetilde{h}_{0}^{(0)} & =\mathrm{i} \int_{\mathbf{q}}(\mathbf{n} \cdot \mathbf{q}) G_{q} H_{\mathbf{q}}^{(0)} e^{\mathrm{iq} \cdot \mathbf{R}^{(0)}} \\
& =-R_{0} \int_{0}^{\infty} d q\left(\frac{q}{q^{2}+\beta}\right) J_{1}^{2}\left(q R_{0}\right) .
\end{aligned}
$$

Applying formula (B.4) to (B.6), we can confirm that (B.6) agrees with (B.2).

\section{Derivation of (68)}

Here, we give the detailed derivation of (68). We first derive $\delta \mathbf{h}_{1,1}^{(0)}$. Up to the first order of the deviations, $\delta \mathbf{h}_{1,1}^{(0)}$ is decomposed as

$$
\begin{gathered}
\delta \mathbf{h}_{1,1}^{(0)}=\frac{\mathrm{i}}{\Omega} \int_{\mathbf{q}} \int d \omega(\mathbf{v} \cdot \mathbf{q}) G_{q}^{2} H_{\mathbf{q}} e^{\mathrm{i} \mathbf{q} \cdot \mathbf{R}} \mathbf{R} \\
-\frac{\mathrm{i}}{\Omega} \int_{\mathbf{q}} \int d \omega(\mathbf{v} \cdot \mathbf{q}) G_{q}^{2} H_{\mathbf{q}}^{(0)} e^{\mathrm{i} \mathbf{q} \cdot \mathbf{R}_{0}} \mathbf{R}_{0} \\
\sim \frac{\mathrm{i}}{\Omega} \int_{\mathbf{q}} \int d \phi_{r}(\mathbf{v} \cdot \mathbf{q}) G_{q}^{2} H_{\mathbf{q}}^{(1)} e^{\mathrm{i} \mathbf{q} \cdot \mathbf{R}_{0}} R_{0}^{2} \mathbf{e}_{r} \\
-\frac{1}{\Omega} \int_{\mathbf{q}} \int d \phi_{r}(\mathbf{v} \cdot \mathbf{q})\left(\mathbf{q} \cdot \delta \mathbf{R}\left(\phi_{r}\right)\right) \\
\times G_{q}^{2} H_{\mathbf{q}}^{(0)} e^{\mathrm{i} \mathbf{q} \cdot \mathbf{R}_{0}} R_{0}^{2} \mathbf{e}_{r} \\
+\frac{2 \mathrm{i}}{\Omega} \int_{\mathbf{q}} \int d \phi_{r}(\mathbf{v} \cdot \mathbf{q}) G_{q}^{2} H_{\mathbf{q}}^{(0)} e^{\mathrm{iq} \cdot \mathbf{R}_{0}} \delta R\left(\phi_{r}\right) R_{0} \mathbf{e}_{r} \\
\equiv \boldsymbol{\Phi}_{1}+\boldsymbol{\Phi}_{2}+\boldsymbol{\Phi}_{3},
\end{gathered}
$$

where $\delta R$ and $H_{\mathbf{q}}$ are given in (25) and (31), respectively.

Substituting $H_{\mathbf{q}}^{(1)}$ into $\Phi_{1}$, the $\alpha(\alpha=1,2)$ component $\Phi_{1(\alpha)}$ is obtained as

$$
\Phi_{1(\alpha)}=\frac{R_{0}}{2} \int d q q^{2} G_{q}^{2} J_{1} J_{2} \sum_{\beta} S_{\alpha \beta} v_{\beta} \equiv-a_{1}^{*} \sum_{\beta} S_{\alpha \beta} v_{\beta} .
$$

Substituting $\delta \mathbf{R}\left(\phi_{r}\right)$ and $H_{\mathbf{q}}^{(0)}$ into $\Phi_{2}, \Phi_{2(\alpha)}$ is obtained as

$$
\begin{aligned}
& \Phi_{2(\alpha)} \\
& =\left[\frac{R_{0}}{4} \int d q q^{2} G_{q}^{2} J_{1} J_{2}-\frac{R_{0}}{4} \int d q q^{2} G_{q}^{2} J_{0} J_{1}\right] \sum_{\beta} S_{\alpha \beta} v_{\beta} .
\end{aligned}
$$

Substituting $\delta R\left(\phi_{r}\right)$ and $H_{\mathbf{q}}^{(0)}$ into $\Phi_{3}, \Phi_{3(\alpha)}$ is obtained as

$$
\Phi_{3(\alpha)}=-\int d q q G_{q}^{2} J_{1}^{2} \sum_{\beta} S_{\alpha \beta} v_{\beta} .
$$

Using the formula for the Bessel function $\left(J_{n-1}(z)-\right.$ $\left.J_{n+1}(z)\right) / 2=J_{n+1}(z)-n J_{n}(z) / z,\left(\Phi_{2(\alpha)}+\Phi_{3(\alpha)}\right)$ becomes

$$
\begin{aligned}
& \left(\Phi_{2(\alpha)}+\Phi_{3(\alpha)}\right) \\
& =\left[\frac{R_{0}}{2} \int d q q^{2} G_{q}^{2} J_{1} J_{2}-\frac{3}{2} \int d q q G_{q}^{2} J_{1}^{2}\right] \sum_{\beta} S_{\alpha \beta} v_{\beta} \\
& \quad \equiv-a_{2}^{*} \sum_{\beta} S_{\alpha \beta} v_{\beta} .
\end{aligned}
$$

$a_{1}^{*}$ and $a_{2}^{*}$ are the same as those of the result in [16]. 
Next, we derive $\delta \widetilde{\mathbf{h}}_{1,1}^{(0)}$. Up to the first order of the deviations, $\delta \widetilde{\mathbf{h}}_{1,1}^{(0)}$ is decomposed as

$$
\begin{aligned}
& \delta \widetilde{\mathbf{h}}_{1,1}^{(0)}=\frac{-1}{\Omega} \int_{\mathbf{q}} \int d \omega(\mathbf{n} \cdot \mathbf{q})(\mathbf{v} \cdot \mathbf{q}) G_{q}^{2} H_{\mathbf{q}} e^{\mathrm{i} \mathbf{q} \cdot \mathbf{R}} \mathbf{R} \\
& +\frac{1}{\Omega} \int_{\mathbf{q}} \int d \omega(\mathbf{n} \cdot \mathbf{q})(\mathbf{v} \cdot \mathbf{q}) G_{q}^{2} H_{\mathbf{q}}^{(0)} e^{\mathrm{i} \mathbf{q} \cdot \mathbf{R}_{0}} \mathbf{R}_{0} \\
& \sim \frac{-2}{\Omega} \int_{\mathbf{q}} \int d \phi_{r}(\mathbf{n} \cdot \mathbf{q})(\mathbf{v} \cdot \mathbf{q}) G_{q}^{2} H_{\mathbf{q}}^{(0)} e^{\mathrm{i} \mathbf{q} \cdot \mathbf{R}_{0}} R_{0} \delta R\left(\phi_{r}\right) \mathbf{e}_{r} \\
& -\frac{1}{\Omega} \int_{\mathbf{q}} \int d \phi_{r}(\mathbf{n} \cdot \mathbf{q})(\mathbf{v} \cdot \mathbf{q}) G_{q}^{2} H_{\mathbf{q}}^{(1)} e^{\mathrm{i} \mathbf{q} \cdot \mathbf{R}_{0}} R_{0}^{2} \mathbf{e}_{r} \\
& -\frac{1}{\Omega} \int_{\mathbf{q}} \int d \phi_{r}(\mathbf{n} \cdot \mathbf{q})(\mathbf{v} \cdot \mathbf{q})\left(\mathrm{i} \mathbf{q} \cdot \delta \mathbf{R}\left(\phi_{r}\right)\right) \\
& \times G_{q}^{2} H_{\mathbf{q}}^{(0)} e^{\mathrm{i} \mathbf{q} \cdot \mathbf{R}_{0}} R_{0}^{2} \mathbf{e}_{r} \\
& +\frac{1}{\Omega} \int_{\mathbf{q}} \int d \phi_{r}(\mathbf{v} \cdot \mathbf{q}) q \delta R^{\prime}\left(\phi_{r}\right) \sin \left(\phi_{q}-\phi_{r}\right) \\
& \times G_{q}^{2} H_{\mathbf{q}}^{(0)} e^{\mathrm{i} \mathbf{q} \cdot \mathbf{R}_{0}} R_{0} \mathbf{e}_{r} \\
& \equiv \Psi_{1}+\Psi_{2}+\Psi_{3}+\Psi_{4} \cdot
\end{aligned}
$$

Substituting $H_{\mathbf{q}}^{(0)}$ and $\delta R\left(\phi_{r}\right)$ into $\Psi_{1}$, the $\alpha(\alpha=1,2)$ component $\Psi_{1(\alpha)}$ is obtained as

$$
\Psi_{1(\alpha)}=\int d q q G_{q}^{2}\left(J_{2}-J_{0}\right) J_{1} \sum_{\beta} S_{\alpha \beta} v_{\beta} .
$$

Substituting $H_{\mathbf{q}}^{(1)}$ into $\Psi_{2}, \Psi_{2(\alpha)}$ is obtained as

$$
\Psi_{2(\alpha)}=-\frac{R_{0}}{4} \int d q q^{2} G_{q}^{2}\left(J_{2}-J_{0}\right) J_{2} \sum_{\beta} S_{\alpha \beta} v_{\beta} .
$$

Substituting $H_{\mathbf{q}}^{(0)}$ and $\delta \mathbf{R}$ into $\Psi_{3}, \Psi_{3(\alpha)}$ is obtained as

$$
\Psi_{3(\alpha)}=\frac{R_{0}}{8} \int d q q^{3} G_{q}^{2}\left(3 J_{1}-J_{2}\right) J_{1} \sum_{\beta} S_{\alpha \beta} v_{\beta} .
$$

Substituting $H_{\mathbf{q}}^{(0)}$ and $\delta R^{\prime}\left(\phi_{r}\right)$ into $\Psi_{4}, \Psi_{4(\alpha)}$ is obtained as

$$
\Psi_{4(\alpha)}=\frac{3}{4} \int d q q^{2} G_{q}^{2} J_{0} J_{1} \sum_{\beta} S_{\alpha \beta} v_{\beta} .
$$

In the main text, $\left(\Psi_{3(\alpha)}+\Psi_{4(\alpha)}\right)$ is expressed as

$$
\begin{aligned}
& \left(\Psi_{3(\alpha)}+\Psi_{4(\alpha)}\right) \\
& \quad=\int d q q^{2} G_{q}^{2}\left[\frac{R_{0}}{8} q\left(3 J_{1}-J_{2}\right) J_{1}+\frac{3}{4} J_{0} J_{1}\right] \sum_{\beta} S_{\alpha \beta} v_{\beta} \\
& \quad \equiv-\widetilde{a}_{1}^{*} \sum_{\beta} S_{\alpha \beta} v_{\beta},
\end{aligned}
$$

and $\left(\Psi_{1(\alpha)}+\Psi_{2(\alpha)}\right)$ is expressed as

$$
\begin{aligned}
& \left(\Psi_{1(\alpha)}+\Psi_{2(\alpha)}\right) \\
& \quad=2 \int d q q G_{q}^{2}\left[\frac{1}{4} R_{0} q J_{2}-J_{1}\right] \frac{\partial J_{1}}{\partial\left(q R_{0}\right)} \sum_{\beta} S_{\alpha \beta} v_{\beta} \\
& \quad \equiv-\widetilde{a}_{2}^{*} \sum_{\beta} S_{\alpha \beta} v_{\beta},
\end{aligned}
$$

where we used $J_{n}^{\prime}(z)=\left(J_{n-1}-J_{n+1}\right) / 2$ as the formula for the Bessel function.

\section{Expansions of $\delta h$ and $\delta \widetilde{h}$ in (75) and (76)}

The expansions of $\delta h$ and $\delta \tilde{h}$ in (75) and (76) are

$$
\begin{aligned}
\delta h_{0} & =-\sum_{n} D_{n} c_{n} e^{\mathrm{i} n \phi_{r}}, \\
\delta h_{1,1} & =-\frac{v_{-}}{2} \sum_{n} A_{n} c_{n} e^{\mathrm{i}(n+1) \phi_{r}}-\frac{v_{+}}{2} \sum_{n} B_{n} c_{n} e^{\mathrm{i}(n-1) \phi_{r}}, \\
\delta h_{1,2} & =-\sum_{n} E_{n} \dot{c}_{n} e^{\mathrm{i} n \phi_{r}}, \\
\delta h_{2,2} & =-\frac{G_{0}}{2}|\mathbf{v}|^{2}+\frac{G_{1}}{4}\left(v_{-}^{2} e^{2 \mathrm{i} \phi_{r}}+v_{+}^{2} e^{-2 \mathrm{i} \phi_{r}}\right) \\
& +\sum_{n} c_{n} e^{\mathrm{i} n \phi_{r}}\left[v_{-}^{2} e^{2 \mathrm{i} \phi_{r}} T_{n, 1}-2|\mathbf{v}|^{2} T_{n, 2}+v_{+}^{2} e^{-2 \mathrm{i} \phi_{r}} T_{n, 3}\right] \\
& +\sum_{n} c_{n} e^{\mathrm{i} n \phi_{r}}\left[\left(v_{-}^{2} e^{2 \mathrm{i} \phi_{r}}+v_{+}^{2} e^{-2 \mathrm{i} \phi_{r}}\right) V_{1}+|\mathbf{v}|^{2} V_{2}\right],
\end{aligned}
$$

$$
\cdots=\cdots \text {, }
$$

$$
\begin{aligned}
& \delta \widetilde{h}_{0}=-\sum_{n} \widetilde{D}_{n} c_{n} e^{\mathrm{i} n \phi_{r}}, \\
& \delta \widetilde{h}_{1,1}=-\frac{v_{-}}{2} \sum_{n}\left(\widetilde{A}_{n}+n \widetilde{A}^{*}\right) c_{n} e^{\mathrm{i}(n+1) \phi_{r}} \\
& -\frac{v_{+}}{2} \sum_{n}\left(\widetilde{B}_{n}-n \widetilde{A}^{*}\right) c_{n} e^{\mathrm{i}(n-1) \phi_{r}}, \\
& \delta \widetilde{h}_{1,2}=-\sum_{n} \widetilde{E}_{n} \dot{c}_{n} e^{\mathrm{i} i \phi_{r}}, \\
& \delta \widetilde{h}_{2,2}=\sum_{n} \widetilde{S}^{*} c_{n} n\left(v_{+}^{2} e^{-2 \mathrm{i} \phi_{r}}-v_{-}^{2} e^{2 \mathrm{i} \phi_{r}}\right) e^{\mathrm{i} n \phi_{r}} \\
& +\left[-\frac{\widetilde{G}_{0}}{2}|\mathbf{v}|^{2}+\frac{\widetilde{G}_{1}}{4}\left(v_{-}^{2} e^{2 \mathrm{i} \phi_{r}}+v_{+}^{2} e^{-2 \mathrm{i} \phi_{r}}\right)\right] \\
& +\sum_{n} c_{n} e^{\mathrm{i} n \phi_{r}}\left[v_{-}^{2} e^{2 \mathrm{i} \phi_{r}} \widetilde{T}_{n, 1}-2|\mathbf{v}|^{2} \widetilde{T}_{n, 2}+\mathrm{v}_{+}^{2} e^{-2 \mathrm{i} \phi_{r}} \widetilde{T}_{n, 3}\right] \\
& +\sum_{n} c_{n} e^{\mathrm{i} n \phi_{r}}\left[\left(v_{-}^{2} e^{2 \mathrm{i} \phi_{r}}+v_{+}^{2} e^{-2 \mathrm{i} \phi_{r}}\right) \widetilde{V}_{1}+|\mathbf{v}|^{2} \widetilde{V}_{2}\right], \\
& \ldots=\ldots,
\end{aligned}
$$

where $v_{ \pm}=v_{1} \pm \mathrm{i} v_{2}$ and all the coefficients in (D.1) and (D.2) are given in Appendix E. In order to calculate the time 
evolution of $c_{n}, e^{-\mathrm{i} n \phi_{r}}$ is multiplied to both sides of eq. (74), and integration is performed over $\phi_{r}$ in the range $0 \leq \phi_{r} \leq 2 \pi$. Using the periodicity of trigonometric functions, we obtain the time evolution equation of $c_{n}$. As the tensors $\mathbf{S}$ and $\mathbf{U}$ are linear combinations of $c_{ \pm n}$, we can derive their time evolution equations.

\section{E. Coefficients in (D.1) and (D.2)}

We give the coefficients in (D.1) and (D.2) below. By using $F(i, j, k, l)$ and $F_{d}(i, j, k, l)$, they are expressed as

$$
\begin{aligned}
& A_{n}=R_{0}\left[F(2,2, n, n+1)+F_{d}(2,2,1,1)\right] \text {, } \\
& B_{n}=R_{0}\left[-F(2,2, n, n-1)+F_{d}(2,2,1,1)\right], \\
& D_{n}=R_{0}[F(1,1,1,1)-F(1,1, n, n)], \\
& E_{n}=R_{0} F(1,2, n, n), \\
& G_{n}=R_{0} F(2,3, n, n+1), \\
& G_{1}^{s}=R_{0} F(4,5,1,2), \\
& T_{n, 1}=\frac{R_{0}}{4} F(3,3, n, n+2), \\
& T_{n, 2}=\frac{R_{0}}{4} F(3,3, n, n) \text {, } \\
& T_{n, 3}=\frac{R_{0}}{4} F(3,3, n, n-2), \\
& V_{1}=\frac{R_{0}}{4} F_{d}(3,3,1,2), \\
& V_{2}=\frac{R_{0}}{2} F(3,3,1,1) \text {, } \\
& \widetilde{A}_{n}=R_{0}\left[\frac{1}{4}(F(3,2,1,3)-3 F(3,2,1,1))\right. \\
& \left.+F_{d}(3,2, n, n+1)\right] \text {, } \\
& \widetilde{A}^{*}=\frac{1}{2}[F(2,2,0,1)+F(2,2,1,2)], \\
& \widetilde{B}_{n}=R_{0}\left[\frac{1}{4}(F(3,2,1,3)-3 F(3,2,1,1))\right. \\
& \left.-F_{d}(3,2, n, n-1)\right] \text {, } \\
& \widetilde{D}_{n}=R_{0}\left[F_{d}(2,1,1,1)-F_{d}(2,1, n, n)\right], \\
& \widetilde{E}_{n}=R_{0} F_{d}(2,2, n, n), \\
& \widetilde{G}_{0}=R_{0} F_{d}(3,3,1,0), \\
& \widetilde{G}_{1}=R_{0} F_{d}(3,3,1,2), \\
& \widetilde{G}_{1}^{s}=R_{0} F_{d}(5,5,1,2), \\
& \widetilde{S}^{*}=-\frac{1}{8}[F(3,3,1,1)+F(3,3,1,3)],
\end{aligned}
$$

$$
\begin{aligned}
& \widetilde{T}_{n, 1}=\frac{R_{0}}{4} F_{d}(4,3, n, n+2), \\
& \widetilde{T}_{n, 2}=\frac{R_{0}}{4} F_{d}(4,3, n, n), \\
& \widetilde{T}_{n, 3}=\frac{R_{0}}{4} F_{d}(4,3, n, n-2), \\
& \widetilde{V}_{1}=\frac{R_{0}}{16}[F(4,3,0,1)-2 F(4,3,1,2)+F(4,3,1,4)], \\
& \widetilde{V}_{2}=\frac{R_{0}}{2} F_{d}(4,3,1,1) .
\end{aligned}
$$

\section{Conflicts of Interest}

The author declares that there are no conflicts of interest.

\section{References}

[1] J. Elgeti, R. G. Winkler, and G. Gompper, "Physics of microswimmers-single particle motion and collective behavior: a review," Reports on Progress in Physics, vol. 78, no. 5, Article ID 056601, 2015.

[2] K. Kroy, D. Chakraborty, and F. Cichos, "Hot microswimmers," The European Physical Journal Special Topics, vol. 225, no. 11-12, pp. 2207-2225, 2016.

[3] A. Geiseler, P. Hänggi, and F. Marchesoni, "Self-Polarizing Microswimmers in Active Density Waves," Scientific Reports, vol. 7, Article ID 41884, 2017.

[4] Y.-J. Chen and K. Yoshikawa, "Interface dynamics under nonequilibrium conditions: From a self-propelled droplet to dynamic pattern evolution," European Physical Journal E, vol. 34, no. 4, Article ID i2011-11038-3, 2011.

[5] N. Yoshinaga, K. H. Nagai, Y. Sumino, and H. Kitahata, "Drift instability in the motion of a fluid droplet with a chemically reactive surface driven by Marangoni flow," Physical Review E: Statistical, Nonlinear, and Soft Matter Physics, vol. 86, no. 1, Article ID 016108, 2012.

[6] S. Yabunaka, T. Ohta, and N. Yoshinaga, "Self-propelled motion of a fluid droplet under chemical reaction," The Journal of Chemical Physics, vol. 136, no. 7, Article ID 074904, 2012.

[7] M. Tarama, A. M. Menzel, B. Ten Hagen, R. Wittkowski, T. Ohta, and H. Löwen, "Dynamics of a deformable active particle under shear flow," The Journal of Chemical Physics, vol. 139, no. 10, Article ID 104906, 2013.

[8] M. Tarama, A. M. Menzel, and H. Löwen, "Deformable microswimmer in a swirl: Capturing and scattering dynamics," Physical Review E: Statistical, Nonlinear, and Soft Matter Physics, vol. 90, no. 3, Article ID 032907, 2014.

[9] Y.-J. Chen, Y. Nagamine, and K. Yoshikawa, "Self-propelled motion of a droplet induced by Marangoni-driven spreading," Physical Review E: Statistical, Nonlinear, and Soft Matter Physics, vol. 80, no. 1, Article ID 016303, 2009.

[10] S. Thutupalli, R. Seemann, and S. Herminghaus, "Swarming behavior of simple model squirmers," New Journal of Physics, vol. 13, Article ID 073021, 2011.

[11] K. H. Nagai, F. Takabatake, Y. Sumino, H. Kitahata, M. Ichikawa, and N. Yoshinaga, "Rotational motion of a droplet induced by interfacial tension," Physical Review E: Statistical, Nonlinear, and Soft Matter Physics, vol. 87, no. 1, Article ID 013009, 2013. 
[12] S. Tanaka, Y. Sogabe, and S. Nakata, "Spontaneous change in trajectory patterns of a self-propelled oil droplet at the airsurfactant solution interface," Physical Review E: Statistical, Nonlinear, and Soft Matter Physics, vol. 91, no. 3, Article ID 032406, 2015.

[13] M. Tarama, Y. Itino, A. M. Menzel, and T. Ohta, "Individual and collective dynamics of self-propelled soft particles," The European Physical Journal Special Topics, vol. 223, no. 1, pp. 121139, 2014.

[14] T. Ohta, "Dynamics of deformable active particles," Journal of the Physical Society of Japan, vol. 86, no. 7, Article ID 072001, 2017.

[15] T. Ohta, "Pulse dynamics in a reaction-diffusion system," Physica D: Nonlinear Phenomena, vol. 151, no. 1, pp. 61-72, 2001.

[16] T. Ohta, T. Ohkuma, and K. Shitara, "Deformation of a selfpropelled domain in an excitable reaction-diffusion system," Physical Review E: Statistical, Nonlinear, and Soft Matter Physics, vol. 80, no. 5, Article ID 056203, 2009.

[17] K. Shitara, T. Hiraiwa, and T. Ohta, "Deformable self-propelled domain in an excitable reaction-diffusion system in three dimensions," Physical Review E: Statistical, Nonlinear, and Soft Matter Physics, vol. 83, no. 6, Article ID 066208, 2011.

[18] A. Nishikimi, H. Fukuhara, W. Su et al., "Sequential regulation of DOCK2 dynamics by two phospholipids during neutrophil chemotaxis," Science, vol. 324, no. 5925, pp. 384-387, 2009.

[19] E. F. Keller and L. A. Segel, "Initiation of slime mold aggregation viewed as an instability," Journal of Theoretical Biology, vol. 26, no. 3, pp. 399-415, 1970.

[20] J. Song and D. Kim, “Three-dimensional chemotaxis model for a crawling neutrophil," Physical Review E: Statistical, Nonlinear, and Soft Matter Physics, vol. 82, no. 5, Article ID 051902, 2010.

[21] J. Song and D. Kim, "Computational kinetic study of chemotactic cell migration," Journal of Applied Physics, vol. 114, no. 15, Article ID 154701, 2013.

[22] L. Zhang, J. Song, and D. Kim, "A study on the interfacial effect on cancer-cell invasion," Journal of Applied Physics, vol. 109, no. 7, Article ID 074702, 2011.

[23] L. Zhang, S. Kang, and D. Kim, "Computational analysis of the effects of matrix-degrading enzymes on cancer-cell invasion," Journal of Applied Physics, vol. 112, no. 12, Article ID 124704, 2012.

[24] L. M. Sander and T. S. Deisboeck, "Growth patterns of microscopic brain tumors," Physical Review E: Statistical, Nonlinear, and Soft Matter Physics, vol. 66, no. 5, Article ID 051901, 2002.

[25] W. G. Tharp, R. Yadav, D. Irimia et al., "Neutrophil chemorepulsion in defined interleukin-8 gradients in vitro and in vivo," Journal of Leukocyte Biology, vol. 79, no. 3, pp. 539-554, 2006.

[26] C. W. Frevert, G. Boggy, T. M. Keenan, and A. Folch, "Measurement of cell migration in response to an evolving radial chemokine gradient triggered by a microvalve," Lab on a Chip, vol. 6, no. 7, pp. 849-856, 2006.

[27] T. Lämmermann, "In the eye of the neutrophil swarmnavigation signals that bring neutrophils together in inflamed and infected tissues," Journal of Leukocyte Biology, vol. 100, no. 1, pp. 55-63, 2016.

[28] S. Kawaguchi and M. Mimura, "Synergistic effect of two inhibitors on one activator in a reaction-diffusion system," Physical Review E: Statistical, Nonlinear, and Soft Matter Physics, vol. 77, no. 4, Article ID 046201, 2008.

[29] S. Kawaguchi, "Chemotaxis-growth under the influence of lateral inhibition in a three-component reaction-diffusion system," Nonlinearity, vol. 24, no. 4, pp. 1011-1031, 2011.
[30] T. Ohta, M. Mimura, and R. Kobayashi, "Higher-dimensional localized patterns in excitable media," Physica D: Nonlinear Phenomena, vol. 34, no. 1-2, pp. 115-144, 1989.

[31] M. Mimura and T. Tsujikawa, "Aggregating pattern dynamics in a chemotaxis model including growth," Physica A: Statistical Mechanics and its Applications, vol. 230, no. 3-4, pp. 499-543, 1996.

[32] K. Krischer and A. Mikhailov, "Bifurcation to traveling spots in reaction-diffusion systems," Physical Review Letters, vol. 73, no. 23, pp. 3165-3168, 1994.

[33] X.-Y. Chen, "Dynamics of interfaces in reaction diffusion systems," Hiroshima Mathematical Journal, vol. 21, no. 1, pp. 4783, 1991.

[34] P. G. de Gennes and J. Prost, The Physics of Liquid Crystals, Oxford University Press, Oxford, UK, 2nd edition, 1995.

[35] L. G. Fel, "Tetrahedral symmetry in nematic liquid crystals," Physical Review E: Statistical, Nonlinear, and Soft Matter Physics, vol. 52, no. 1, pp. 702-717, 1995.

[36] H. R. Brand, H. Pleiner, and P. E. Cladis, "Tetrahedratic crosscouplings: Novel physics for banana liquid crystals," Physica A: Statistical Mechanics and its Applications, vol. 351, no. 2-4, pp. 189-197, 2005.

[37] T. Hiraiwa, M. Y. Matsuo, T. Ohkuma, T. Ohta, and M. Sano, "Dynamics of a deformable self-propelled domain," EPL (Europhysics Letters), vol. 91, no. 2, Article ID 20001, 2010.

[38] E. Meron, "Pattern formation in excitable media," Physics Reports, vol. 218, no. 1, pp. 1-66, 1992.

[39] E. O. Budrene and H. C. Berg, "Complex patterns formed by motile cells of Escherichia coli," Nature, vol. 349, no. 6310, pp. 630-633, 1991.

[40] M. Kronlage, J. Song, L. Sorokin et al., "Autocrine purinergic receptor signaling is essential for macrophage chemotaxis," Science Signaling, vol. 3, no. 132, p. ra55, 2010.

[41] M. Mimura, H. Sakaguchi, and M. Matsushita, "Reactiondiffusion modelling of bacterial colony patterns," Physica A: Statistical Mechanics and its Applications, vol. 282, no. 1, pp. 283303, 2000.

[42] D. A. Kessler and H. Levine, "Pattern formation in Dictyostelium via the dynamics of cooperative biological entities," Physical Review E: Statistical, Nonlinear, and Soft Matter Physics, vol. 48, no. 6, pp. 4801-4804, 1993.

[43] J. Rinzel and D. Terman, "Propagation phenomena in a bistable reaction-diffusion system," SIAM Journal on Applied Mathematics, vol. 42, no. 5, pp. 1111-1137, 1982.

[44] I. S. Gradshteyn and I. M. Ryzhik, Table of Integrals, Series, and Products, Academic Press, 6th edition, 2000. 


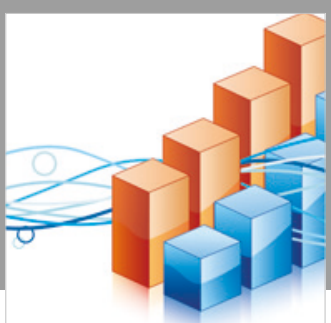

Advances in

Operations Research

\section{-n-m}
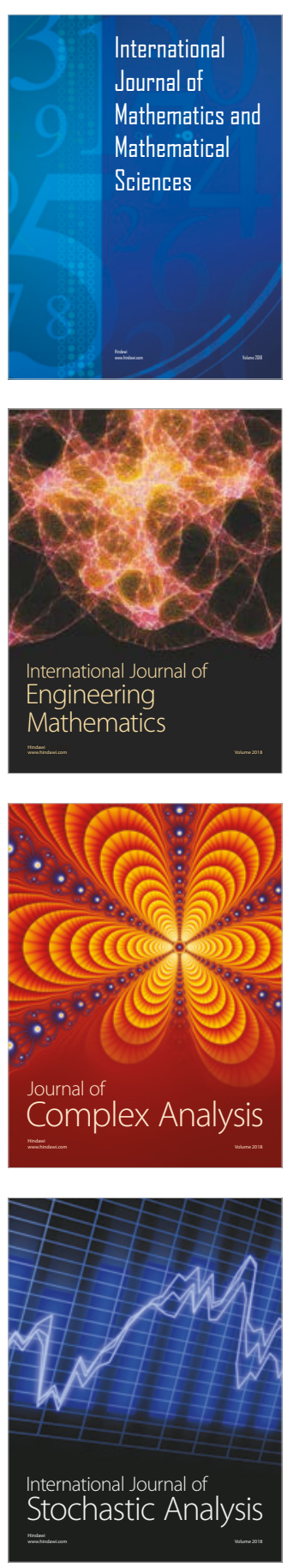
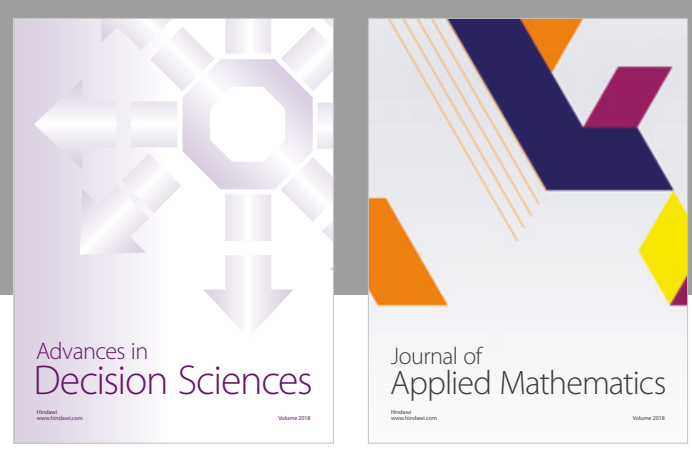

Journal of

Applied Mathematics
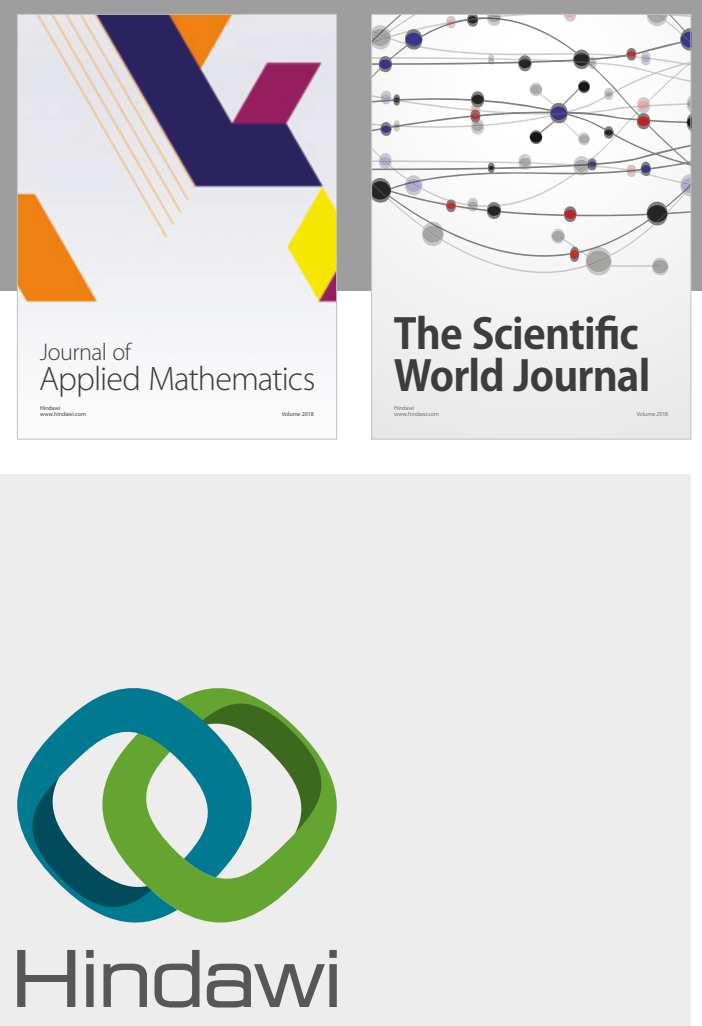

Submit your manuscripts at

www.hindawi.com

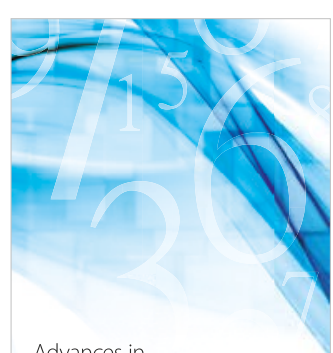

Advances in
Numerical Analysis
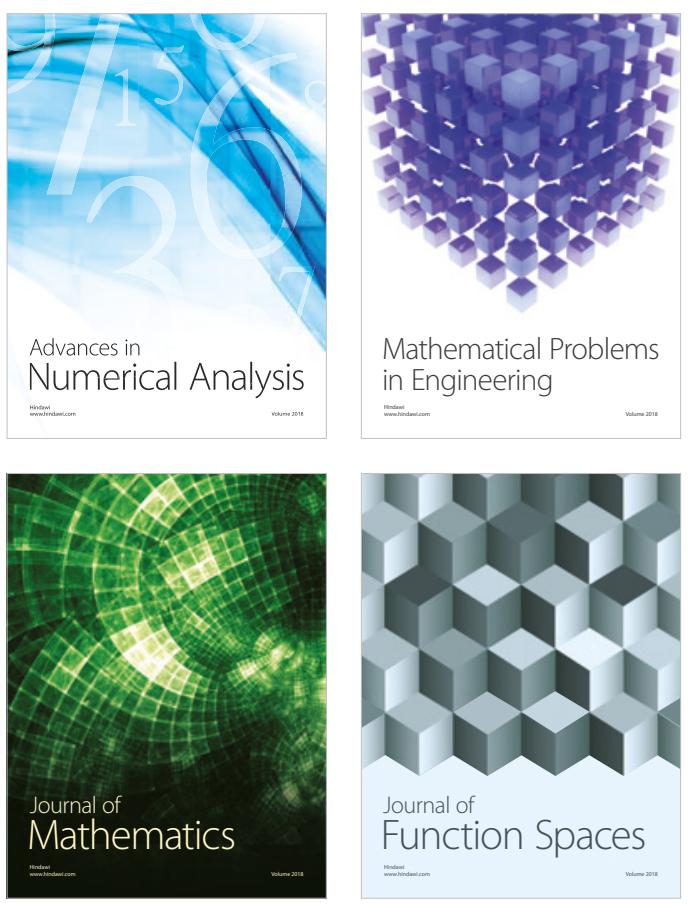

Mathematical Problems in Engineering

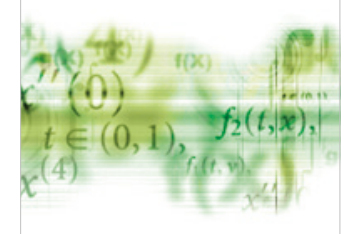

International Journal of

Differential Equations

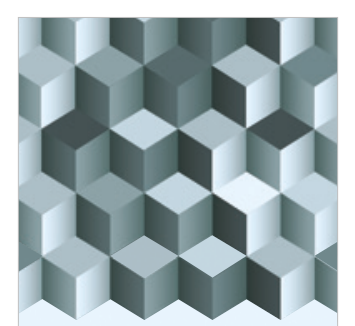

Journal of

Function Spaces

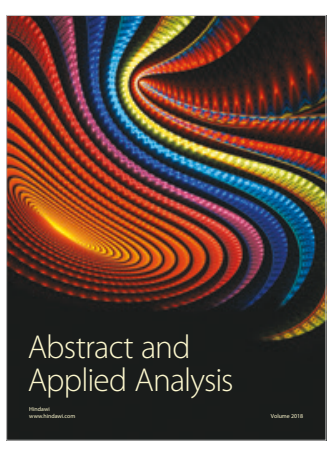

The Scientific

World Journal

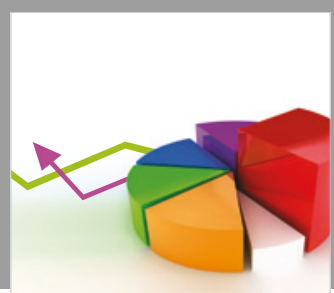

Journal of

Probability and Statistics
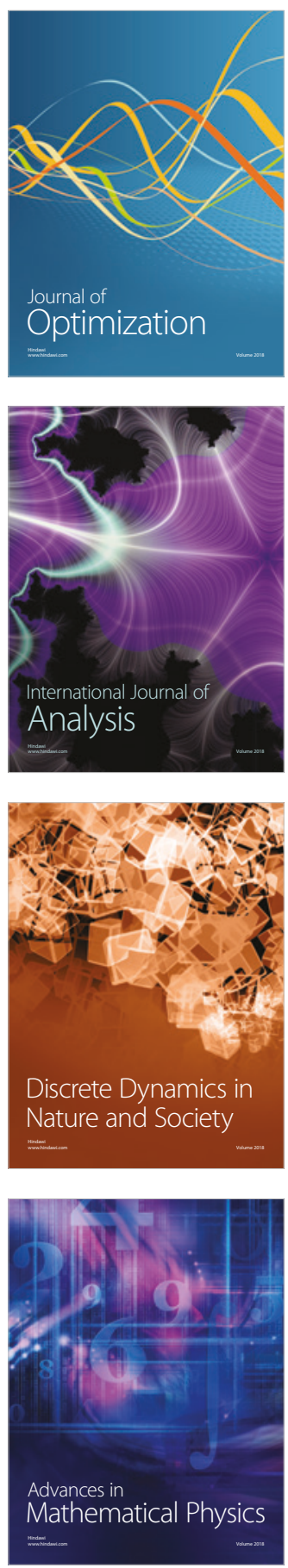